\title{
SYSTEMS OF HYPERBOLIC CONSERVATION LAWS WITH MEMORY
}

\author{
CLEOPATRA CHRISTOFOROU
}

\begin{abstract}
Global weak solutions of bounded variation to systems of balance laws with non-local source are constructed by the method of vanishing viscosity. Suitable dissipativeness assumptions are imposed on the source terms to assure convergence of the method. Under these hypotheses, the total variation remains uniformly bounded and integrable in time, the vanishing viscosity solutions are uniformly stable in $L^{1}$ with respect to the initial data and converge to equilibrium as $t \rightarrow \infty$. The motivation to study these systems is the observation that conservations laws with fading memory can be written in such form under appropriate conditions on the flux.
\end{abstract}

\section{INTRODUCTION}

Systems of conservation laws in one-space dimension are equations of the form

$$
\partial_{t} U(t, x)+\partial_{x} S(t, x)=0,
$$

where $x \in \mathbb{R}$ and $U, S \in \mathbb{R}^{n}$. Such equations are called conservation laws because in classical physics, conservation of mass, momentum and energy of media are governed by equations of this type.

In this context, $U(t, x)$ determines the state of the medium at the point $(t, x)$ and $S(t, x)$ is the flux of $U$. In general, materials are identified by constitutive relations which describe how the flux is determined by the state vector $U$. The medium is called elastic if $S(t, x)$ is determined by the value of state $U$ at $(t, x)$ i.e. (1.1) reduces to

$$
\partial_{t} U(t, x)+\partial_{x} F(U(t, x))=0,
$$

where $F: \mathbb{R}^{n} \rightarrow \mathbb{R}^{n}$ is a given smooth function. The Cauchy problem to (1.2) has been studied in great detail. When $F$ is nonlinear, the profile of solutions starting out from smooth initial data gets progressively steeper and eventually breaks in finite time, by developing jump discontinuities which propagate on as shock waves. This raises the need to deal with weak solutions, cf. [18]. The global existence of weak solutions was first established by the "random choice method" in the celebrated paper of Glimm [16]. Expositions of the current state of the theory may be found in the books $[4,9,26,28]$.

The model (1.2) fails in the presence of viscosity and relaxation phenomena. In such circumstances, the flux $S(x, t)$ depends also on the past history $U(x, \tau)$ for

1991 Mathematics Subject Classification. 35L65.

Key words and phrases. Hyperbolic systems, fading memory term, vanishing viscosity, viscoelasticity, heat flow, convolution. 
$\tau<t$ and we say that the material has memory. An important class of media of this type are materials with fading memory for which the constitutive relations yield

$$
U_{t}+F(U(x, t))_{x}+\int_{0}^{t} k(t-\tau) G(U(x, \tau))_{x} d \tau=0
$$

where $F, G: \mathbb{R}^{n} \rightarrow \mathbb{R}^{n}$ are smooth functions and $k:[0, \infty) \rightarrow \mathbb{M}_{n \times n}$ is a smooth kernel integrable over $[0, \infty)$. Extensive studies have been conducted to contrast the behavior of elastic media with the behavior of media with fading memory. The results show that when the kernel satisfies appropriate conditions motivated by physical considerations, the influence of the memory term in (1.3) is dissipative. Consequently, global smooth solutions to (1.3) exist for given small and smooth initial data and tend to the equilibrium as $t \rightarrow \infty$, in constract to the situation with elastic media that classical solutions in general break down in finite time. However, when the initial data is large, the destabilizing action of nonlinearity of the flux function $F$ prevails over the damping, and solutions to (1.3) break down in a finite time. (cf. Renardy-Hrusa-Nohel [25] and the references therein).

It worths mentioning that there are several results in classical theory in the literature on nonlinear viscoelasticity and heat flow that can be represented by a second order equation

$$
u_{t t}=\sigma\left(u_{x}\right)_{x}+\int_{0}^{t} a(t-\tau) \psi\left(u_{x}(\tau)\right)_{x} d \tau+f(t, x),
$$

which is equivalent to system (1.3). E.g., see [12, 15, 19, 20, 21, 22, 23, 27, 29, 17].

Global weak solutions have also been constructed for special equations of the form (1.3) mainly in $L^{\infty}$ by the method of compensated compactness. See [10, 24, 6]. However, it is still an open problem to establish globally defined weak solutions of bounded variation $(B V)$ of $(1.3)$.

As suggested by Dafermos [13], systems (1.3) are equivalent to hyperbolic systems of the form

$$
U_{t}+A(U) U_{x}+g(U)=H(t) \bar{U}-\int_{0}^{t} K(t-\tau) U(\tau) d \tau,
$$

for special $F$ and $G$, where $A, H, K$ are $n \times n$ matrices, $g \in \mathbb{R}^{n}$ and $\bar{U} \in \mathbb{R}^{n}$ is the initial data. The motivation is to view (1.3) as a Volterra equation and by means of resolvent kernel and integration by parts to reduce the memory term to the above non-local source term. This observation is due to MacCamy [19, 20] and later employed by Dafermos [10,11] and Nohel-Rogers-Tzavaras [24] in order to get $L^{\infty}$ weak solutions. The advantage of studying system (1.5) is that it is easier to extract the damping effect from the source $g(U)$ than from the fading memory term in (1.3). It should be noted that the same approach was followed to apply the method of compensated compactness in the aforementioned papers. However, system (1.5) is written in a more general form than the one treated in the past. Memory damping is similar to frictional damping, therefore, the damping may not be always balanced and this would be reflected on the $g(U)$ term in the sense of Dafermos-Hsiao [14], i.e. the diagonal dominance condition. In such cases, we may attempt to transfer part of the damping and achieve a good balance. For more details on this subject and some examples see [13].

The aim of this paper is to construct global weak solutions of BV to (1.5) as suggested by Dafermos, whence to (1.3). Here, we employ the vanishing viscosity 
method that was first established for systems of conservation laws in a spectacular way by Bianchini and Bressan [3]. This method was extended to systems of conservation laws with dissipative source

$$
U_{t}+A(U) U_{x}+g(U)=0
$$

by Chistoforou [7]. The dissipativeness assumptions imposed to (1.6) are the same as the ones suggested by Dafermos-Hsiao [14], who employed Glimm's scheme [16]. Our goal is to prove that the vanishing viscosity approximations are globally defined with uniform bounded total variation independently of the viscosity parameter. Therefore, as the viscosity coefficient tends to zero we can pass to the limit and get an admissible weak solution to (1.5) in $B V$. Because of the damping effect, we are actually able to show that the total variation as a function of time is integrable over $[0, \infty)$, hence the weak solution constructed by this method converges to the equilibrium as $t \rightarrow \infty$. Thus, we validate the results of the classical theory in the general context of weak solutions.

Recently, Chen and Christoforou [5] studied the non-local conservation law with fading memory, that is the scalar equation of the form (1.3) with $F=G$ and showed that there exists a unique global weak solution by the method of vanishing viscosity. They also established the limit to the local conservation law when there is a relaxation kernel. We remark that the results in this paper extend the project [5] to systems. It would be interesting to study the case of a relaxation kernel for systems (1.3) as well. However, this is a difficult task since the techniques of vanishing viscosity for systems are still new and complex. Nevertheless, we are able to recover the limit of solutions to

$$
U_{t}+A(U) U_{x}+g(U)=H(t) \bar{U},
$$

as $K \rightarrow 0$ pointwise a.e.

The strategy of the paper is the following: In Section 2, we state the assumptions and the theorem. In section 3, we give a roadmap of the proof. The proof is presented in Section 3-8 and follows closely the fundamental work of Bianchini and Bressan and the additional techniques of Christoforou to deal with source terms. Last, the limit as the $K$ tends to zero pointwise almost everywhere to the local conservation law (1.7) is discussed in the end of Section 2.

\section{Assumptions And Theorem}

In view of the analysis in the introduction, we consider the following Cauchy problem in one-space dimension

$$
\begin{aligned}
U_{t}+A(U) U_{x}+g(U) & =H(t) \bar{U}-\int_{0}^{t} K(t-\tau) U(\tau) d \tau \\
U(0, x) & =\bar{U}(x), \quad x \in \mathbb{R} .
\end{aligned}
$$

Here $A: \mathbb{R}^{n} \mapsto M_{n \times n}, g: \mathbb{R}^{n} \mapsto \mathbb{R}^{n}$, and $H, K:[0, \infty) \rightarrow M_{n \times n}(\mathbb{R})$, are given sufficiently smooth functions and $\bar{U} \in \mathbb{R}^{n}$. The subscripts $t$ and $x$ denote partial derivatives. We assume that the system is strictly hyperbolic, i.e. $A(U)$ has $\mathrm{n}$ real distinct eigenvalues

$$
\lambda_{1}(U)<\lambda_{2}(U)<\ldots<\lambda_{n}(U)
$$

and thereby $\mathrm{n}$ linearly independent right eigenvectors $r_{i}(u), i=1, \ldots, n$. Note that we do not require system (2.1) to be conservative. 
In this project, we would like to construct global weak solutions to (2.1)-(2.2) of bounded variation via the vanishing viscosity method, namely we consider the viscous hyperbolic system

$$
U_{t}^{\varepsilon}+A\left(U^{\varepsilon}\right) U_{x}^{\varepsilon}+g\left(U^{\varepsilon}\right)=H(t) \bar{U}-\int_{0}^{t} K(t-\tau) U^{\varepsilon}(\tau) d \tau+\varepsilon U_{x x}^{\varepsilon}
$$

and obtain globally defined viscous approximations $U^{\varepsilon}$ that are uniformly stable and converge in $L_{l o c}^{1}$ to the weak solution $U$ to (2.1)-(2.2). For arbitrary source terms in (2.1), only local weak solutions exist even if the initial data are small. In order to prove global existence, we need to impose conditions on the hyperbolic system that induce dissipative mechanisms. Namely, let $U^{*}$ be a constant equilibrium to (2.1), then the assumptions are the following:

\section{Assumptions. A}

(1) $\tilde{B} \doteq R\left(U^{*}\right)^{-1} B R\left(U^{*}\right)$ is strictly column-diagonally dominant, i.e. there exists a positive constant $\beta>0$, such that

$$
\tilde{B}_{i i}-\sum_{j \neq i}\left|\tilde{B}_{j i}\right|>\beta>0
$$

for every $i=1, \ldots, n$.

(2) $\tilde{K}(s) \doteq R\left(U^{*}\right)^{-1} K(s) R\left(U^{*}\right) \in L^{1}[0,+\infty)$ is absolutely column dominated by $\tilde{B}$, i.e. there exists a positive constant $\kappa>0$, such that

$$
\int_{0}^{+\infty} \sum_{j=1}^{n}\left|\tilde{K}_{j i}(s)\right| d s<\kappa, \quad \text { for all } i=1, \ldots, n
$$

and

$$
0 \leq \kappa<\beta
$$

(3) $H(\cdot) \in L^{1}[0,+\infty)$.

Some comments on the above assumptions: We recall that Dafermos and Hsiao [14] treated systems (1.6) by the random choice method and proved global existence under Assumption (1). Also, Christoforou [7] recently established the vanishing viscosity method for systems (1.6) under the same assumption. In other words, Assumption (1) induces the dissipation on the system via the source $g$. Moreover, we impose Assumption (2) to assure that the convolution $K * u$ term can be treated as a lower order perturbation in terms of $g$. Last, Assuption (3) is needed since we require $L^{1}$ bounds. For symmetry, we define $\tilde{H}(t) \doteq R\left(U^{*}\right)^{-1} H(t) R\left(U^{*}\right)$.

The principal results are stated in the following theorem.

Theorem 1. Consider the Cauchy problem

$$
\begin{gathered}
U_{t}^{\varepsilon}+A\left(U^{\varepsilon}\right) U_{x}^{\varepsilon}+g\left(U^{\varepsilon}\right)=H(t) \bar{U}-\int_{0}^{t} K(t-\tau) U^{\varepsilon}(\tau, x) d \tau+\varepsilon U_{x x}^{\varepsilon} \\
U^{\varepsilon}(0, x)=\bar{U}(x) .
\end{gathered}
$$

Assume that the matrices $A(U)$ have real distinct eigenvalues $\lambda_{1}(U)<\lambda_{2}(U)<$ $\ldots<\lambda_{n}(U)$ and Assumptions A hold. There exists a constant $\delta_{0}>0$ such that if $\bar{U}-U^{*} \in L^{1}$ and

$$
T V\{\bar{U}\}<\delta_{0},
$$


then for each $\varepsilon>0$ the Cauchy problem (2.5)-(2.6) has a unique solution $U^{\varepsilon}$, defined for all $t \geq 0$, that satisfies

$$
T V\left\{U^{\varepsilon}(t, \cdot)\right\}+\int_{0}^{t} T V\left\{U^{\varepsilon}(s, \cdot)\right\} d s \leq C T V\{\bar{U}\},
$$

where $C$ is a positive constant that is independent of $t$ and $\varepsilon$. Moreover, the solutions to (2.5) are stable $L^{1}$ with respect to the initial data; if $V^{\varepsilon}$ is another solution of (2.5) with initial data $\bar{V}$, then

$$
\left\|U^{\varepsilon}(t)-V^{\varepsilon}(t)\right\|_{L^{1}}+\int_{0}^{t}\left\|U^{\varepsilon}(\tau)-V^{\varepsilon}(\tau)\right\|_{L^{1}} d \tau \leq L\|\bar{U}-\bar{V}\|_{L^{1}} .
$$

Furthermore, the continuous dependence property with respect to time holds, i.e.

$$
\left\|U^{\varepsilon}(t)-U^{\varepsilon}(s)\right\|_{L^{1}} \leq L^{\prime}(|t-s|+\sqrt{\varepsilon}|\sqrt{t}-\sqrt{s}|),
$$

for $t, s>0$. Finally, as $\varepsilon \rightarrow 0, U^{\varepsilon}$ converges in $L_{l o c}^{1}$ to a function $U$, which is the admissible weak solution $U$ of (2.1)-(2.2), when the system is in conservation form, $A=D F$.

The integrability of the total variation is induced by the dissipativeness Assumptions (1)-(2) and in particular the requirement $\kappa<\beta$. Recall that in [7], when $H=K=0$, the total variation is exponentially decaying in time. Thus, the above result is an expected generalization of the result in [7]. In view of the discussion in the introduction, this theorem implies the global existence of weak solutions of bounded variation to hyperbolic systems of conservation laws with fading memory (1.3) when (1.3) can be written in the form (2.1).

As already mentioned, we follow closely the fundamental work of Bianchini and Bressan [3] and the techniques of Christoforou [7] to prove Theorem 1. To begin with, by rescaling the coordinates, $t \sim t / \varepsilon, x \sim x / \varepsilon$, system (2.5)-(2.6) reduces to (2.11)

$$
\begin{gathered}
U_{t}+A(U) U_{x}-U_{x x}+\varepsilon g(U)=\varepsilon H(\varepsilon t) U_{0}-\varepsilon^{2} \int_{0}^{t} K(\varepsilon(t-\tau)) U(\tau) d \tau+U_{x x}, \\
U(0, x)=U_{0}(x)=\bar{U}(\varepsilon x) .
\end{gathered}
$$

The total variation of the initial data $U_{0}^{\varepsilon}$ does not change with $\varepsilon$, while the $L^{1}$ norm does. Our goal is to establish a bound

$$
T V\{U(t, \cdot)\}+\varepsilon \int_{0}^{t} T V\{U(s, \cdot)\} d s \leq C T V\{\bar{U}\}
$$

for all times $t \geq 0$, with $C$ depending solely on the total variation of $\bar{U}$ and not on $\left\|U_{0}\right\|_{L^{1}}$

In order to establish the stability estimate (2.9) in Theorem 1, we shall also work with the linearized evolution equation which governs an infinitesimal perturbation $Z$ of $U$ :

$Z_{t}+A(U) Z_{x}+\varepsilon D g(U) Z-Z_{x x}+(Z \bullet A(U)) U_{x}=\varepsilon H(\varepsilon t) Z_{0}-\varepsilon^{2} \int_{0}^{t} K(\varepsilon(t-\tau)) Z(\tau) d \tau$, and establish a bound of the form

$$
\|Z(t)\|_{L^{1}}+\varepsilon \int_{0}^{t}\|Z(s)\| d s \leq L\|Z(0)\|_{L^{1}} .
$$


Having (2.12) and (2.13), we can complete the proof.

The proof of the theorem is presented in the following sections. The structure of the paper is: In Section 3, we outline the proof for the convenience of the reader. In Section 4, we prove (2.12) and (2.14) over a time interval of length $\mathcal{O}\left(\delta_{0}^{-2}\right)$ by using standard parabolic estimates. We also obtain $L^{1}$ bounds of higher order derivatives of $U_{x}$ and $Z$. In Sections $5-7$, we extend the validity of these estimates up to $t=\infty$, by using the hyperbolic structure of the system. The reason of this two-step approach is that the parabolic estimates apply even when the derivatives of the initial data are large, but are only valid on a finite time interval, whose length depends on total variation of initial data; whereas, the hyperbolic estimates are valid for all times, but require initial values with small derivatives. Finally, in Section 8 by rescaling the coordinates backwards, we complete the proof of Theorem 1.

Now, lets return to the original hyperbolic system (2.1) for a moment. Since the existence of weak solutions to (2.1)-(2.2) has been established by Theorem 1, we raise the question of letting the kernel $K$ tend to zero pointwise or equivalently $\kappa \rightarrow 0$. To emphasize the dependence of solutions to $(2.1)-(2.2)$ on $K$, we denote the admissible weak solution constructed by the method of vanishing viscosity by $U^{\kappa}$ and assume $\kappa \in\left(0, \kappa_{0}\right)$.

Remark 2. Suppose that the hypotheses of Theorem 1 hold and $\kappa \in\left(0, \kappa_{0}\right)$. Denote by $U^{\kappa}(t, x)$ the weak solution to (2.1)-(2.2) obtained by the vanishing viscosity method. Moreover, assume that the kernel $K$ of the non-local source term in (2.1) tends to 0 pointwise a.e. Then, $U^{\kappa}$ converges in $L_{l o c}^{1}$ to an admissible weak solution $U$ of the Cauchy problem

$$
\begin{gathered}
U_{t}+A(U) U_{x}+g(U)=H(t) \bar{U}, \\
U(0, x)=\bar{U}(x), \quad x \in \mathbb{R} .
\end{gathered}
$$

The validity of the above remark is due to the observation that all estimates (2.8)-(2.10) are independent not only of $\varepsilon$, but also of $1 / \kappa$. Indeed, all calculations in the following sections show that as the viscosity parameter $\varepsilon$ vanishes

$$
T V\left\{U^{\kappa}(t, \cdot)\right\}+\alpha \int_{0}^{t} T V\left\{U^{\kappa}(s, \cdot)\right\} d s \leq C T V\{\bar{U}\},
$$

where $\alpha-(\beta-\kappa)=\mathcal{O}(1) \delta_{0}$ and $C>0$ depends on $\kappa_{0}$ and $1 /(\beta-\kappa)$. Therefore, we can extract a convergent subsequence $U^{\kappa_{j}}$ that converges to the admissible weak solution of (2.15)-(2.16).

It should be noted that if $H \equiv 0$, then in this way we recover the weak solution to (1.6) as constructed in [7]. Observe that in such a case $\alpha \approx \beta$, which is the same exponential decay rate of the total variation as shown in [7]. Thus, the estimates to $(2.1)-(2.2)$ reduce to the corresponding ones in [7] as $\kappa \rightarrow 0$.

\section{Outline OF THE PROOF}

The proof is carried out in two steps. Using parabolic estimates, we first obtain the local existence of solutions to (2.11) and prove the desired bounds (2.12) and (2.14) for $t \in[0, \hat{t}]$, where $\hat{t}=\mathcal{O}\left(\delta_{0}^{-2}\right)$. We also show that if $(2.12)$ and $(2.14)$ hold for $t \geq \hat{t}$, then similar $L^{1}$-bounds hold in on higher-order derivatives of $U$ and $Z$. 
Having this result, we are able to decompose the gradient

$$
U_{x}=\sum_{i=1}^{n} V_{i} \tilde{r}_{i}
$$

into a sum of gradients of viscous traveling waves selected by a center manifold technique. This critical idea of Bianchini and Bressan in [3] helps to get a sharp estimate in $\left\|U_{x}(t)\right\|_{L^{1}}$. We modify the decomposition for the time derivative $U_{t}$ in order to correct the speed of the viscous traveling waves to our setting, in other words to take care the source terms in a way similar to Christoforou in [7], i.e.

$$
U_{t}+\varepsilon \Delta(U, t, \varepsilon)=\sum_{i=1}^{n}\left(W_{i}-\lambda_{i}^{*} V_{i}\right) \tilde{r}_{i}
$$

where

$$
\Delta:=g(U)-H(\varepsilon t) U_{0}+\varepsilon \int_{0}^{t} K(\varepsilon(t-\tau)) U(\tau) d \tau
$$

and $\lambda_{i}^{*}=\lambda_{i}\left(U^{*}\right)$. It remains to study the evolution of each component $V_{i}$ and $W_{i}$. We show that $(V, W)$ satisfy a $2 n \times 2 n$ coupled-system of viscous balance laws of similar type to (2.11). More precisely, we have

$$
\begin{array}{r}
\left(\begin{array}{c}
V \\
W
\end{array}\right)_{t}+\left[\left(\begin{array}{cc}
\Lambda & 0 \\
0 & \Lambda
\end{array}\right)\left(\begin{array}{c}
V \\
W
\end{array}\right)\right]_{x}-\left(\begin{array}{c}
V \\
W
\end{array}\right)_{x x}+\varepsilon\left(\begin{array}{ll}
B^{1} & B^{\sharp} \\
B^{b} & B^{2}
\end{array}\right)\left(\begin{array}{c}
V \\
W
\end{array}\right)=\varepsilon\left(\begin{array}{ll}
H^{1} & H^{\sharp} \\
H^{b} & H^{2}
\end{array}\right)\left(\begin{array}{c}
V_{0} \\
W_{0}
\end{array}\right) \\
-\varepsilon^{2} \int_{0}^{t}\left(\begin{array}{cc}
K^{1} & K^{\sharp} \\
K^{b} & K^{2}
\end{array}\right)\left(\begin{array}{c}
V(\tau) \\
W(\tau)
\end{array}\right) d \tau+\left(\begin{array}{c}
\Phi \\
\Psi
\end{array}\right)
\end{array}
$$

where $\Lambda=\operatorname{diag}\left(\tilde{\lambda}_{i}\right)$ is a diagonal matrix with $\tilde{\lambda}_{i} \approx \lambda_{i}$, the matrix

$$
\mathcal{B} \doteq\left(\begin{array}{ll}
B^{1} & B^{\sharp} \\
B^{b} & B^{2}
\end{array}\right)
$$

is diagonally dominant and satisfies

$$
\left\|\left(\begin{array}{cc}
B^{1} & B^{\sharp} \\
B^{b} & B^{2}
\end{array}\right)-\left(\begin{array}{cc}
\tilde{B} & 0 \\
0 & \tilde{B}
\end{array}\right)\right\|_{\infty}=\mathcal{O}(1) \delta_{0},
$$

where $\tilde{B}$ is defined by Assumption (2). Also, the matrix

$$
\mathcal{H} \doteq\left(\begin{array}{ll}
H^{1} & H^{\sharp} \\
H^{\mathrm{b}} & H^{2}
\end{array}\right)
$$

satisfies

$$
\varepsilon \sup _{x}|\mathcal{H}| \in L^{1}\left(M_{2 n \times 2 n} ;[0,+\infty)\right), \quad \varepsilon\|\mathcal{H}\|_{L^{1}}=\|\tilde{H}\|_{L^{1}}+\mathcal{O}(1) \delta_{0}
$$

Last,

$$
\mathcal{K} \doteq\left(\begin{array}{ll}
K^{1} & K^{\sharp} \\
K^{b} & K^{2}
\end{array}\right)
$$

is absolutely column dominated by $\mathcal{B}$ for sufficiently small $\delta_{0}$. More precisely,

$$
\left\|\left(\begin{array}{cc}
K^{1} & K^{\sharp} \\
K^{b} & K^{2}
\end{array}\right)-\left(\begin{array}{cc}
\tilde{K} & 0 \\
0 & \tilde{K}
\end{array}\right)\right\|_{\infty}+\varepsilon \int_{0}^{\infty}\left\|\left(\begin{array}{cc}
K^{1} & K^{\sharp} \\
K^{b} & K^{2}
\end{array}\right)-\left(\begin{array}{cc}
\tilde{K} & 0 \\
0 & \tilde{K}
\end{array}\right)\right\|_{\infty} d s=\mathcal{O}(1) \delta_{0},
$$


and

$$
\varepsilon \sum_{j=1}^{n} \int_{0}^{+\infty}\left|K_{j i}^{1}(\varepsilon s)\right|+\left|K_{j i}^{b}(\varepsilon s)\right| d s=\kappa+\mathcal{O}(1) \delta_{0} .
$$

Similarly for the matrices $K^{2}$ and $K^{\sharp}$. By $\mathcal{O}(1)$, we denote a universal constant independent of $\varepsilon$ and $t$. It worths noting that the $\varepsilon$ factor in the above estimates is induced by the rescaling of coordinates.

It suffices to show that all $\left|\Phi_{i}\right|$ and $\left|\Psi_{i}\right|$ are integrable over the half plane $\{t>$ $\hat{t}, x \in \mathbb{R}\}$. We analyze the form of the various source terms $\Phi$ and $\Psi$ that can be regarded as the result of interactions between viscous waves. To achieve this, we employ various functionals that were introduced in [3] as well as supplementary functionals that control source terms as in [7]. In this way, we finally estimate the integral

$$
\int_{\hat{t}}^{\infty} \int \sum_{i}\left(\left|\Phi_{i}(t, x)\right|+\left|\Psi_{i}(t, x)\right|\right) d x d t=\mathcal{O}(1) \delta_{0}^{2}
$$

and this yields the desired a priori bound on $\left\|U_{x}(t, \cdot)\right\|_{L^{1}}$.

This was a summary of the main steps in the proof of the following lemma which plays a central role in the proof of BV bounds (2.12).

Lemma 3.1. Let $U$ be a solution of (2.11) such that for $t \in[\hat{t}, T], U$ satisfies

$$
\left\|U_{x}(t)\right\|_{L^{1}}+\varepsilon \int_{\hat{t}}^{t}\left\|U_{x}(s)\right\|_{L^{1}} d s \leq \delta_{0} .
$$

Assume that the vectors $\Phi$ and $\Psi$ that appear in the source of (3.2) satisfy

$$
\int_{\hat{t}}^{T} \int \sum_{i}\left(\left|\Phi_{i}(t, x)\right|+\left|\Psi_{i}(t, x)\right|\right) d x d t \leq \delta_{0},
$$

then the following estimate holds

$$
\int_{\hat{t}}^{T} \int \sum_{i=1}^{n}\left(\left|\Phi_{i}(t, x)\right|+\left|\Psi_{i}(t, x)\right|\right) d x d t=\mathcal{O}(1) \delta_{0}^{2},
$$

for sufficiently small $\delta_{0}$.

Now, we proceed to complete the proof of the uniform BV bounds. Consider any initial data $\bar{U}: \mathbb{R} \longmapsto \mathbb{R}^{n}$, with

$$
T V\{\bar{U}\} \leq \frac{\delta_{0}\left(1+\|\tilde{H}\|_{L^{1}}\right)^{-1}}{8 \sqrt{n} k\left(1+\|H\|_{L^{1}}\right)}, \quad \quad \lim _{x \rightarrow-\infty} \bar{U}(x)=U^{*} \in K,
$$

for some appropriate constant $k$. By parabolic estimates, the solution $U$ to (2.11) exists on an initial time interval $[0, \hat{t}]$, satisfying the bound

$$
\left\|U_{x}(\hat{t})\right\|_{L^{1}}+\varepsilon \int_{0}^{\hat{t}}\left\|U_{x}(s)\right\|_{L^{1}} d s \leq \frac{1}{4 \sqrt{n}} \delta_{0}
$$

and can be prolonged in time as long as its total variation remains small. Define the time

$$
T \doteq \sup \left\{\tau ; \sum_{i} \int_{\hat{t}}^{\tau} \int\left(\left|\Phi_{i}(t, x)\right|+\left|\Psi_{i}(t, x)\right|\right) d x d t \leq \frac{\delta_{0}}{4}\right\} .
$$


We claim that $T=\infty$. Indeed, if $T<\infty$, then, for $t \in[\hat{t}, T]$, the properties of system (3.2) imply

$$
\sum_{i}\left\|V_{i}\right\|_{t}+\varepsilon(\beta-\mu) \sum_{i}\left\|V_{i}\right\| \leq \varepsilon \sup _{x}(\mathcal{H})\left\|V_{0}\right\|+\sum_{i}\left\|\Phi_{i}\right\|
$$

$$
-\varepsilon^{2} \sum_{i} \int_{0}^{t}\left[K_{i i}^{1}(\varepsilon(t-\tau))-\sum_{j \neq i}\left|K_{j i}^{1}(\varepsilon(t-\tau))\right|-\sum_{j}\left|K_{j i}^{b}(\varepsilon(t-\tau))\right|\right]\left\|V_{i}(\tau)\right\| d \tau
$$

where $\|\cdot\|$ denotes the $L^{1}$-norm and $\mu>0$ an arbitrarily small positive constant that is $\mu=\mathcal{O}(1)\left(\delta_{0}\right)$. Let $\|V\|=\sum_{i}\left\|V_{i}\right\|_{L^{1}}$. Integrating with respect to time, changing the order of integration and using (3.4), we get

$$
\begin{aligned}
\|V(t)\|+\varepsilon(\beta-\mu) \int_{\hat{t}}^{t}\|V(\tau)\| d \tau & \leq\|V(\hat{t})\|+\varepsilon\left\|\sup _{x} \mathcal{H}\right\|_{L^{1}}\left\|V_{0}\right\|+\int_{\hat{t}}^{t}\|\Phi(\tau)\| d \tau \\
& +\varepsilon(\kappa+\mu) \int_{0}^{t}\|V(\tau)\| d \tau
\end{aligned}
$$

Equivalently,

$$
\begin{aligned}
\|V(t)\|+\varepsilon(\beta-\kappa-2 \mu) \int_{\hat{t}}^{t}\|V(\tau)\| d \tau \leq & \|V(\hat{t})\|+\varepsilon(\kappa+\mu) \int_{0}^{\hat{t}}\|V(\tau)\| d \tau \\
& +\left(\|\tilde{H}\|_{L^{1}}+\mathcal{O}(1) \delta_{0}\right)\left\|V_{0}\right\|+\int_{\hat{t}}^{t}\|\Phi(\tau)\| d \tau
\end{aligned}
$$

for sufficiently small $\delta_{0}$. Since $\beta-\kappa-2 \mu>0$ for sufficiently small $\delta_{0}$, without loss of generality we assume $\beta-\kappa-2 \mu=1$. Otherwise, we can always scale all multiples of $\delta_{0}$ to achieve the same contradiction argument. Hence for all $t \in[\hat{t}, T]$, by (3.9), (3.8), (3.3) and (3.10), we conclude

$$
\begin{aligned}
& \left\|U_{x}(t)\right\|_{L^{1}}+\varepsilon \int_{\hat{t}}^{t}\left\|U_{x}(t)\right\|_{L^{1}} \\
& \leq\|V(t)\|+\varepsilon \int_{\hat{t}}^{t}\|V(\tau)\| d \tau \\
& \leq 2 \sqrt{n} \frac{\delta_{0}}{4 \sqrt{n}}+\left(\|\tilde{H}\|_{L^{1}}+\mathcal{O}(1) \delta_{0}\right) 2 \sqrt{n} T V\{\bar{U}\}+\frac{\delta_{0}}{4}<\delta_{0}
\end{aligned}
$$

By Lemma 3.1, it follows

$$
\sum_{i} \int_{\hat{t}}^{T} \int\left(\left|\Phi_{i}(t, x)\right|+\left|\Psi_{i}(t, x)\right|\right) d x d t=\mathcal{O}(1) \delta_{0}^{2}<\frac{\delta_{0}}{2}
$$

which leads to a contradiction by the choice of $T$ in (3.10) provided that $\delta_{0}$ is suitably small. Thus, the total variation remains bounded, $T V\{U(t)\}<\delta_{0}$ for all $t \in[\hat{t}, \infty)$, whence the solution $U$ is globally defined.

The proof of Lemma 3.1 will be established by studying carefully the form of the source terms $\Phi_{i}$ and $\Psi_{i}, i=1, \ldots, n$.

The analysis of stability is in Section 7. The proof of (2.14) is a generalization of the above techniques.

\section{Parabolic estimates.}

This section investigates the local existence of solutions to (2.11) and (2.13) and bounds (2.12) and (2.14) over an initial time interval by means of parabolic estimates. 
To begin with, we rewrite system (2.13) as

$$
\begin{aligned}
Z_{t}+A^{*} Z_{x}+\varepsilon D g\left(U^{*}\right) Z-Z_{x x}= & \varepsilon H(\varepsilon t) Z_{0}-\varepsilon^{2} \int_{0}^{t} K(\varepsilon(t-\tau)) Z(\tau) d \tau, \\
& +\left(A^{*}-A(U)\right) Z_{x}-(Z \bullet A(U)) U_{x} \\
& +\varepsilon\left(D g\left(U^{*}\right)-D g(U)\right) Z
\end{aligned}
$$

where $A^{*} \doteq A\left(U^{*}\right)$ and $D$ is the gradient operator with respect to the state vector $U$. Also, $s \bullet \Phi$ denotes the derivative of $\Phi$ in the direction of $s$, i.e. $\nabla_{s} \Phi$.

We quote the following lemma of [7], which provides us with $L^{1}$ bounds on the Green's kernel of the principal part of (4.1).

Lemma 4.1. Under the hypotheses of Theorem 1, for all $t>0$, the Green's kernel $G$ of the parabolic system

$$
w_{t}+A^{*} w_{x}-w_{x x}+\varepsilon D g\left(U^{*}\right) w=0
$$

satisfies the bounds

$$
\|G(t)\|_{L^{1}}+\varepsilon \int_{0}^{t}\|G(s)\|_{L^{1}} d s \leq k, \quad\left\|G_{x}(t)\right\|_{L^{1}} \leq \frac{k}{\sqrt{t}}, \quad\left\|G_{x x}(t)\right\|_{L^{1}} \leq \frac{k}{t},
$$

for some appropriate constant $k$.

To be precise, the above lemma in [7] states that the above $L^{1}$ bounds have exponential decay, i.e. $\|G(t)\|_{L^{1}} \leq k e^{-\varepsilon \beta t}$, etc, where $\beta$ is the constant induced by the diagonal dominance condition, see Assumption (1).

Here, we define some useful constants:

$$
\begin{gathered}
\hat{t} \doteq\left(\frac{1}{400 k \alpha \delta_{0}}\left(1+\beta^{-1}\right)\right)^{2}, \quad \varepsilon_{0} \doteq k^{2} \alpha \delta_{0}^{2}<<k^{2} \alpha \delta_{0}, \\
\alpha \doteq \sup _{u}\left(\|D A\|_{\infty}+\left\|D^{2} A\right\|_{\infty}+\left\|D^{2} g\right\|_{\infty}\right)+\|K(t)\|_{L^{1}[0, \infty)}+\|H(t)\|_{\infty},
\end{gathered}
$$

where $k$ is the constant in $(4.3)$ and $\delta_{0}<<1$.

By means of parabolic arguments, we now establish the following results:

Proposition 4.2. Let $U, Z$ be solutions of systems (2.11), (2.13) respectively, satisfying the bounds

$$
\left\|U_{x}(t)\right\|_{L^{1}}+\varepsilon \int_{0}^{t}\left\|U_{x}(\tau)\right\|_{L^{1}} d \tau \leq \delta_{0}, \quad\|Z(t)\|_{L^{1}}+\varepsilon \int_{0}^{t}\|Z(\tau)\|_{L^{1}} d \tau \leq \delta_{0},
$$

for some constant $0<\delta_{0}<1$ and for all $t \in[0, \hat{t}]$ and $\varepsilon \in\left[0, \varepsilon_{0}\right]$. Then for $t \in[0, \hat{t}]$ the following estimates hold:

$$
\begin{gathered}
\left\|U_{x x}(t)\right\|_{L^{1}},\left\|Z_{x}(t)\right\|_{L^{1}} \leq \frac{2 k \delta_{0}}{t^{1 / 2}}, \\
\left\|U_{x x x}(t)\right\|_{L^{1}},\left\|Z_{x x}(t)\right\|_{L^{1}} \leq \frac{5 k^{2} \delta_{0}}{t}, \\
\left\|U_{x x x}(t)\right\|_{L^{\infty}},\left\|Z_{x x}(t)\right\|_{L^{\infty}} \leq \frac{16 k^{3} \delta_{0}}{t^{3 / 2}},
\end{gathered}
$$

where $k$ is the constant in (4.3). 
Moreover, if the bounds (4.6) hold on a larger interval $[0, T]$, then for all $t \in$ $[\hat{t}, T]$

$$
\begin{aligned}
\left\|U_{x x}(t)\right\|_{L^{1}},\left\|U_{x}(t)\right\|_{L^{\infty}},\left\|Z_{x}(t)\right\|_{L^{1}} & =\mathcal{O}(1) \delta_{0}^{2} \\
\left\|U_{x x x}(t)\right\|_{L^{1}},\left\|U_{x x}(t)\right\|_{L^{\infty}},\left\|Z_{x x}(t)\right\|_{L^{1}} & =\mathcal{O}(1) \delta_{0}^{3} \\
\left\|U_{x x x}(t)\right\|_{L^{\infty}},\left\|Z_{x x}(t)\right\|_{L^{\infty}} & =\mathcal{O}(1) \delta_{0}^{4}
\end{aligned}
$$

Proof. Employing the Green's function $G(t, x)$ and (4.1), the infinitesimal perturbation $Z$ of $U$ can be written as follows

$$
\begin{aligned}
Z(t) & =G(t) * Z(0)+\int_{0}^{t} G(t-s) *\left[\left(A^{*}-A(U)\right) Z_{x}(s)-(Z \bullet A(U)) U_{x}(s)\right] d s \\
& +\varepsilon \int_{0}^{t} G(t-s) * H(\varepsilon s) Z(0) d s+\varepsilon \int_{0}^{t} G(t-s) *\left(D g\left(U^{*}\right)-D g(U)\right) Z(s) d s
\end{aligned}
$$

$$
-\varepsilon^{2} \int_{0}^{t} G(t-s) * \int_{0}^{s} K(\varepsilon(s-\tau)) Z(\tau) d \tau d s,
$$

where $*$ denotes convolution. Differentiating with respect to $x$ yields

$$
\begin{aligned}
& Z_{x}(t)=G_{x}(t) * Z(0)+\int_{0}^{t} G_{x}(t-s) *\left[\left(A^{*}-A(U)\right) Z_{x}(s)-(Z \bullet A(U)) U_{x}(s)\right] d s \\
& \quad+\varepsilon \int_{0}^{t} G_{x}(t-s) * H(\varepsilon s) Z(0) d s+\varepsilon \int_{0}^{t} G_{x}(t-s) *\left(D g\left(U^{*}\right)-D g(U)\right) Z(s) d s
\end{aligned}
$$

$$
-\varepsilon^{2} \int_{0}^{t} G_{x}(t-s) * \int_{0}^{s} K(\varepsilon(s-\tau)) Z(\tau) d \tau d s
$$

Let $\mathcal{T}<\hat{t}$ be the first time at which (4.7) holds as an equality, then we estimate $\left\|Z_{x}(\mathcal{T})\right\|_{L^{1}}$ via (4.14). By (4.6) and Lemma 4.1, we get

$$
\begin{aligned}
\left\|Z_{x}(\mathcal{T})\right\|_{L^{1}} \leq & \left\|G_{x}(\mathcal{T})\right\|_{L^{1}}\|Z(0)\|_{L^{1}}+\int_{0}^{\mathcal{T}}\left\|G_{x}(\mathcal{T}-s)\right\|_{L^{1}}\left[\left\|\left(A^{*}-A(U)\right) Z_{x}(s)\right\|_{L^{1}}\right. \\
& +\left\|(Z \bullet A(U)) U_{x}(s)\right\|_{L^{1}}+\varepsilon\left\|\left(D g\left(U^{*}\right)-D g(U)\right) Z(s)\right\|_{L^{1}} \\
& \left.+\varepsilon|H(\varepsilon s)|\|Z(0)\|_{L^{1}}+\varepsilon^{2} \int_{0}^{s}|K(\varepsilon(s-\tau))|\|Z(\tau)\|_{L^{1}} d \tau\right] d s \\
\leq & \frac{k}{\sqrt{\mathcal{T}}} \delta_{0}+\int_{0}^{\mathcal{T}} \frac{k}{\sqrt{\mathcal{T}-s}}\left[\|D A\|_{L^{\infty}}\left\|U_{x}(s)\right\|_{L^{1}}\left\|Z_{x}(s)\right\|_{L^{1}}\right. \\
& +\|Z(s)\|_{L^{\infty}}\|D A\|_{L^{\infty}}\left\|U_{x}(s)\right\|_{L^{1}}+\varepsilon\left\|D^{2} g\right\|_{L^{\infty}}\left\|U_{x}(s)\right\|_{L^{1}}\|Z(s)\|_{L^{1}} \\
& \left.+\varepsilon\|H\|_{\infty} \delta_{0}+\varepsilon \delta_{0}\|K\|_{L^{1}}\right] d s \\
< & \frac{k \delta_{0}}{\sqrt{\mathcal{T}}}+2\|D A\|_{L^{\infty}} \int_{0}^{\mathcal{T}} \frac{k}{\sqrt{\mathcal{T}-s}} \frac{2 k \delta_{0}}{\sqrt{s}} d s+\varepsilon\left\|D^{2} g\right\|_{L^{\infty}} \delta_{0}^{2} k 2 \sqrt{\mathcal{T}} \\
& +\varepsilon k \delta_{0}\|H\|_{\infty} 2 \sqrt{\mathcal{T}}+\varepsilon k \delta_{0}\|K\|_{L^{1}} 2 \sqrt{\mathcal{T}} \\
< & {\left[\frac{k \delta_{0}}{\sqrt{\mathcal{T}}}+16 k^{2} \alpha \delta_{0}^{2}+\varepsilon k \delta_{0} 2 \sqrt{\mathcal{T}}\left(\left\|D^{2} g\right\|_{L^{\infty}} \delta_{0}+\|H\|_{\infty}+\|K\|_{L^{1}}\right)\right] }
\end{aligned}
$$


For values of $\varepsilon$ in $\left[0, \varepsilon_{0}\right]$, the above estimate simplifies to

$$
\left\|Z_{x}(\mathcal{T})\right\|_{L^{1}}<\left[\frac{k \delta_{0}}{\sqrt{\mathcal{T}}}+\frac{1}{25} \frac{k \delta_{0}}{\sqrt{\mathcal{T}}}+\frac{1}{8 \cdot 10^{4}} \frac{k \delta_{0}}{\sqrt{\mathcal{T}}}\right]<\frac{2 k \delta_{0}}{\sqrt{\mathcal{T}}}
$$

which contradicts the choice of $\mathcal{T}$. Hence the estimate (4.7) holds over the interval $[0, \hat{t}]$. Moreover, a solution of $(4.1)$ is $Z=U_{x}$, hence the same bound holds for $\left\|U_{x}(t)\right\|_{L^{1}}$.

To prove the remaining estimates, we argue in the same manner. We first express $Z_{x x}(t)$ in terms of the Green's kernel $G$ and then estimate $\left\|Z_{x x}(t)\right\|_{L^{1}}$ and $\left\|Z_{x x}(t)\right\|_{L^{\infty}}$ via the above equation.

To prove (4.10)-(4.12) for $t \in[\hat{t}, T]$, apply the same estimates on the time interval $[t-\hat{t}, t]$, whose length is $\hat{t} \approx \delta_{0}^{-2}$.

Similarly, we prove $(2.12)$ and $(2.14)$ over $[0, \hat{t}]$.

Proposition 4.3. Let $U=U(t, x), Z=Z(t, x)$ be solutions of (2.11), (2.13), respectively, such that

$$
T V\{U(0, \cdot)\} \leq \frac{\delta_{0}}{4 k\left(1+\|H\|_{L^{1}(0, \infty)}\right)}, \quad\|Z(0)\|_{L^{1}} \leq \frac{\delta_{0}}{4 k\left(1+\|H\|_{L^{1}(0, \infty)}\right)} .
$$

Then $U, Z$ are well-defined on the whole interval $[0, \hat{t}]$ satisfying the bounds

$$
\left\|U_{x}(t)\right\|_{L^{1}}+\varepsilon \int_{0}^{t}\left\|U_{x}(s)\right\|_{L^{1}} d s \leq \frac{\delta_{0}}{2}, \quad\|Z(t)\|_{L^{1}}+\varepsilon \int_{0}^{t}\|Z(s)\|_{L^{1}} d s \leq \frac{\delta_{0}}{2}
$$

for all $t \in[0, \hat{t}]$.

Proof. The proof is by contradiction. If $\mathcal{T}<\hat{t}$ is the first time at which (4.18) holds as an equality, then by the expression (4.13) for $t=\mathcal{T}$, we get

$$
\begin{aligned}
& \|Z(\mathcal{T})\|_{L^{1}} \leq\|G(\mathcal{T})\|_{L^{1}}\|Z(0)\|_{L^{1}}+\int_{0}^{\mathcal{T}}\|G(\mathcal{T}-s)\|_{L^{1}}\left[\left\|U_{x}(s)\right\|_{L^{1}}\|D A\|_{L^{\infty}}\left\|Z_{x}(s)\right\|_{L^{1}}\right. \\
& \left.\quad+\|Z(s)\|_{L^{\infty}}\|D A\|_{L^{\infty}}\left\|U_{x}(s)\right\|_{L^{1}}+\varepsilon\left\|U_{x}(s)\right\|_{L^{1}}\left\|D^{2} g\right\|_{L^{\infty}}\|Z(s)\|_{L^{1}}\right] d s \\
& \quad+\varepsilon \int_{0}^{\mathcal{T}}\|G(\mathcal{T}-s)\|_{L^{1}}|H(\varepsilon s)|\|Z(0)\|_{L^{1}}
\end{aligned}
$$

$$
+\varepsilon^{2} \int_{0}^{\mathcal{T}}\|G(\mathcal{T}-s)\|_{L^{1}} \int_{0}^{s}|K(\varepsilon(s-\tau))|\|Z(\tau)\| d \tau d s
$$

If one sets $Z=U_{x}$, then the above estimate proves the bound (4.18.1) by a contradiction argument. Having this, we derive the bound (4.18.2) on $Z$ as follows: by 
(4.7), (4.17), (4.18.1) and the above inequality, yields

$$
\begin{aligned}
\|Z(\mathcal{T})\|_{L^{1}} & +\varepsilon \int_{0}^{\mathcal{T}}\|Z(t)\|_{L^{1}} d t \leq k\|Z(0)\|_{L^{1}} \\
& +\int_{0}^{\mathcal{T}} G_{2}(\mathcal{T}-s)\left(2 \frac{\delta_{0}}{2}\|D A\|_{L^{\infty}} \frac{2 k \delta_{0}}{\sqrt{s}}+\varepsilon \frac{\delta_{0}^{2}}{4}\left\|D^{2} g\right\|_{L^{\infty}}\right) d s \\
& +\varepsilon \int_{0}^{\mathcal{T}} G_{2}(\mathcal{T}-s)|H(\varepsilon s)|\|Z(0)\|_{L^{1}} d s \\
& +\varepsilon^{2} \int_{0}^{\mathcal{T}} G_{2}(\mathcal{T}-s) \int_{0}^{s}|K(\varepsilon(s-\tau))|\|Z(\tau)\| d \tau d s
\end{aligned}
$$

where

$$
G_{2}(\mathcal{T}-s) \doteq\|G(\mathcal{T}-s)\|_{L^{1}}+\varepsilon \int_{s}^{\mathcal{T}}\|G(t-s)\|_{L^{1}} d t
$$

It is easy to verify that $\|G(t)\|_{L^{1}}+\varepsilon \int_{0}^{\infty}\|G(s)\|_{L^{1}} d s<\left(1+\frac{1}{\beta}\right)$, using the proof of Lemma 4.1 in [7]. Hence, the above estimate reduces to

$$
\begin{aligned}
\|Z(\mathcal{T})\|_{L^{1}}+\varepsilon \int_{0}^{\mathcal{T}}\|Z(t)\|_{L^{1}} d t & \leq k\left(1+\|H\|_{L^{1}[0, \infty)}\right)\|Z(0)\|_{L^{1}} \\
& +2 k\left(1+\frac{1}{\beta}\right) \delta_{0}^{2}\|D A\|_{L^{\infty}} \sqrt{\mathcal{T}} \\
& +\varepsilon\left(1+\frac{1}{\beta}\right) \frac{\delta_{0}}{2} \mathcal{T}\left[\frac{\delta_{0}}{2}\left\|D^{2} g\right\|_{L^{\infty}}+\|K\|_{L^{1}[0, \infty)}\right] .
\end{aligned}
$$

Consequently,

$$
\|Z(\mathcal{T})\|_{L^{1}}+\varepsilon \int_{0}^{\mathcal{T}}\|Z(t)\|_{L^{1}} d t<\left[\frac{\delta_{0}}{4}+\frac{\delta_{0}}{200}+\frac{\delta_{0}}{8 \cdot 10^{4}}\right]<\frac{\delta_{0}}{2},
$$

for all $\varepsilon \in\left[0, \varepsilon_{0}\right)$. This contradicts the choice of $\mathcal{T}<\hat{t}$. The proof is complete.

\section{Decomposition Along gradients of Viscous traveling WaVEs.}

In this section, we decompose the gradient $U_{x}$ and the time derivative $U_{t}$ pointwise along gradients of viscous traveling waves as first constructed by Bianchini and Bressan [3, 2] by a center manifold argument. The decomposition is similar to that one used in [7]. First, we quote a summary of the results and the main estimates in [3]. For each $i$, we consider the viscous traveling i-waves $U\left(x-\sigma_{i} t\right)$ that are solutions to the system of conservation laws $U_{t}+A(U) U_{x}=U_{x x}$, having speed $\sigma_{i} \approx \lambda_{i}\left(U^{*}\right)$ and corresponding to trajectories of the flow

$$
\begin{aligned}
& \dot{u}=v \\
& \dot{v}=\left(A(u)-\sigma_{i} I\right) v \\
& \dot{\sigma}_{i}=0
\end{aligned}
$$

that lie on the center manifold $\mathcal{C}_{i} \subset \mathbb{R}^{n} \times \mathbb{R}^{n} \times \mathbb{R}$

$$
\mathcal{C}_{i}=\left\{\left(U, V, \sigma_{i}\right): V=V_{i} \tilde{r}_{i},\left|U-U^{*}\right|<\delta,\left|V_{i}\right|<\delta,\left|\sigma_{i}-\lambda_{i}^{*}\right|<\delta\right\}
$$


as described in Section 4 of [3]. The unit vector $\tilde{r}_{i}=\tilde{r}_{i}\left(U, V_{i}, \sigma_{i}\right)$ is defined in a small neighborhood of $\left(U^{*}, 0, \lambda_{i}^{*}\right)$ and tends to $r_{i}^{*} \doteq r_{i}\left(U^{*}\right)$ as $\left(U, V_{i}, \sigma_{i}\right) \rightarrow\left(U^{*}, 0, \lambda_{i}^{*}\right)$. We use the same notation as in $[3,7]$ :

$$
\tilde{r}_{i, U} \doteq \frac{\partial \tilde{r}_{i}}{\partial U}, \quad \tilde{r}_{i, V} \doteq \frac{\partial \tilde{r}_{i}}{\partial V_{i}} \quad \tilde{r}_{i, \sigma} \doteq \frac{\partial \tilde{r}_{i}}{\partial \sigma_{i}}
$$

The following identity plays a key role in controlling the component source terms $\Phi_{i}$ and $\Psi_{i}$ :

$$
\left(A(U)-\tilde{\lambda}_{i} I\right) \tilde{r}_{i}=V_{i}\left(\tilde{r}_{i, U} \tilde{r}_{i}+\left(\tilde{\lambda}_{i}-\sigma_{i}\right) \tilde{r}_{i, V}\right),
$$

where $\tilde{\lambda}_{i}$ is the "generalized eigenvalue" defined as $\tilde{\lambda}_{i} \doteq\left\langle\tilde{r}_{i}, A(U) \tilde{r}_{i}\right\rangle$. This identity is fundamental since it corresponds to $\left(A(U)-\lambda_{i} I\right) r_{i}=0$. By continuity, (5.4) implies

$$
\tilde{r}_{i}\left(U, 0, \sigma_{i}\right)=r_{i}(U),
$$

and $\tilde{\lambda}_{i} \rightarrow \lambda_{i}(U)$ as $V_{i} \rightarrow 0$ and we deduce the following important estimates:

$$
\begin{aligned}
\tilde{r}_{i}\left(U, V_{i}, \sigma_{i}\right)-r_{i}(U) & =\mathcal{O}(1) \cdot V_{i}, & \tilde{r}_{i, \sigma} & =\mathcal{O}(1) \cdot V_{i}, \\
\tilde{r}_{i, U \sigma} & =\mathcal{O}(1) \cdot V_{i} & \tilde{r}_{i, \sigma \sigma} & =\mathcal{O}(1) \cdot V_{i}, \\
\left|\tilde{\lambda}_{i}\left(U, V_{i}, \sigma_{i}\right)-\lambda_{i}(U)\right| & =\mathcal{O}(1) \cdot V_{i}, & \tilde{\lambda}_{i, \sigma} & =\mathcal{O}(1) \cdot V_{i} .
\end{aligned}
$$

Without loss of generality, we can assume that the eigenvectors $r_{i}\left(U^{*}\right)$ form an orthonormal basis, hence

$$
\begin{aligned}
\left\langle\tilde{r}_{i}\left(U, V_{i}, \sigma_{i}\right), \tilde{r}_{j}\left(U, V_{j}, \sigma_{j}\right)\right\rangle & =\delta_{i j}+\mathcal{O}(1) \delta_{0}, \\
\left\langle\tilde{r}_{i}, A(U) \tilde{r}_{j}\right\rangle & =\mathcal{O}(1) \delta_{0} \quad \text { for } i \neq j .
\end{aligned}
$$

These estimates of [3] will be used in the forthcoming sections in order to control the component source terms $\Phi_{i}, \Psi_{i}$.

Now, we employ the above construction to decompose $U_{x}$. Let $U$ be a smooth solution of system of viscous balance laws (2.11). For each point $x$, given $\left(U, U_{x}\right.$, $\left.U_{x x}\right)$, we seek $(V, W) \in \mathbb{R}^{2 n}$ such that

$$
\begin{gathered}
U_{x}=\sum_{i=1}^{n} V_{i} \tilde{r}_{i}\left(U, V_{i}, \sigma_{i}\right) \\
U_{t}+\varepsilon \Delta(U, t, \varepsilon)=\sum_{i=1}^{n}\left(W_{i}-\lambda_{i}^{*} V_{i}\right) \tilde{r}_{i}\left(U, V_{i}, \sigma_{i}\right),
\end{gathered}
$$

while

$$
\Delta:=g(U)-H(\varepsilon t) U_{0}+\varepsilon \int_{0}^{t} K(\varepsilon(t-\tau)) U(\tau) d \tau .
$$

The speed is given by

$$
\sigma_{i}=\lambda_{i}^{*}-\theta\left(\frac{W_{i}}{V_{i}}\right)
$$

where $\theta$ is a cutoff function

$$
\theta(s)=\left\{\begin{array}{ll}
s & |s| \leq \delta_{1} \\
0 & |s| \geq 3 \delta_{1},
\end{array} \quad\left|\theta^{\prime}(s)\right| \leq 1, \quad\left|\theta^{\prime \prime}(s)\right| \leq 4 / \delta_{1} .\right.
$$

It is necessary to insert the cutoff function $\theta$ in order to guarantee that the speed $\sigma_{i}$ remains close to $\lambda_{i}^{*}$ and therefore $\tilde{r}_{i}\left(U, V_{i}, \sigma_{i}\right)$ is well defined. From now on, we use the usual abbreviation $\theta_{i} \doteq \theta\left(\frac{W_{i}}{V_{i}}\right)$. The presence of $\varepsilon$-term in the decomposition of 
$U_{t}$ is crucial, as it corrects the speed of the viscous $i$-traveling waves when passing from the system of conservation laws to the system of balance laws. Observe that the decomposition (5.10) corresponds to viscous traveling waves $U_{i}$ such that

$$
U_{i}(x)=U(x), \quad U_{i}^{\prime}(x)=V_{i} \tilde{r}_{i}, \quad U_{i}^{\prime \prime}=\left(A(U)-\sigma_{i}\right) U_{i} .
$$

Thus, $U_{x}(x)=\sum_{i} U_{i}^{\prime}(x)$ and

$$
U_{x x}(x)=\sum_{i} U_{i}^{\prime \prime}(x)
$$

when the cutoff function $\theta$ is the identity map. For more details, see the calculations in [7]. In other words, when the cutoff is not active, there is also a good fit of the second derivative $U_{x x}$ due to the $\varepsilon$-term in (5.10).

The validity of the decomposition is confirmed by the following lemma.

Lemma 5.1. Assume that $\left|U-U^{*}\right|,\left|U_{x}\right|$ and $\left|U_{x x}\right|$ are sufficiently small. Then there exists a unique solution $(V, W)$ to system $(5.10)$ such that the map $\left(U, U_{x}, U_{x x}\right) \mapsto$ $(V, W)$ is smooth outside the manifolds $\mathcal{N}_{i}=\left\{(V, W) ; V_{i}=W_{i}=0\right\}, i=1, \ldots, n ;$ more precisely it is $\mathcal{C}^{1,1}$, i.e. continuously differentiable with Lipschitz continuous derivatives on a neighborhood of the point $\left(U^{*}, 0,0\right)$.

Proof. Following [7], the map $(V, W) \mapsto \mathcal{G}(U ; V, W)$ defined by

$$
\begin{gathered}
\mathcal{G}: \mathbb{R}^{n} \times \mathbb{R}^{n} \times \mathbb{R}^{n} \mapsto \mathbb{R}^{2 n} \\
(U, V, W) \mapsto \sum_{i=1}^{n} \mathcal{G}_{i}\left(U ; V_{i}, W_{i}\right), \\
\mathcal{G}_{i}\left(U ; V_{i}, W_{i}\right)=\left(\begin{array}{l}
V_{i} \tilde{r}_{i}\left(U, V_{i}, \lambda_{i}^{*}-\theta\left(\frac{W_{i}}{V_{i}}\right)\right) \\
\left(W_{i}-\lambda_{i}^{*} V_{i}\right) \tilde{r}_{i}\left(U, V_{i}, \lambda_{i}^{*}-\theta\left(\frac{W_{i}}{V_{i}}\right)\right)
\end{array}\right),
\end{gathered}
$$

is $\mathcal{C}^{1}$ and invertible in a small neighborhood of $(0,0)$. Thus given $\left(U, U_{x}, U_{x x}\right)$, there exists unique $(V, W)$ such that

$$
\mathcal{G}(U ; V, W)=\left(U_{x}, U_{x x}-A(U) U_{x}\right)=\left(U_{x}, U_{t}+\varepsilon \Delta(U, t, \varepsilon)\right)
$$

and (5.10) holds. Following the details in [7], one can complete the proof.

The following lemma states the bounds on $V$ and $W$ that correspond to the bounds on the $U_{x}, U_{x x}$ and $U_{x x x}$ derived in Section 4.

Lemma 5.2. Assume that bounds (4.6) hold on $[0, T]$, then, the components $V_{i}$, $W_{i}$ in (5.10) satisfy the following estimates

$$
\begin{aligned}
\left\|V_{i}(t)\right\|_{L^{1}},\left\|W_{i}(t)\right\|_{L^{1}}, \varepsilon \int_{0}^{t}\left\|V_{i}(s)\right\|_{L^{1}} d s, \varepsilon \int_{0}^{t}\left\|W_{i}(s)\right\|_{L^{1}} d s & =\mathcal{O}(1) \delta_{0}, \\
\left\|V_{i}(t)\right\|_{L^{\infty}},\left\|W_{i}(t)\right\|_{L^{\infty}},\left\|V_{i, x}(t)\right\|_{L^{1}},\left\|W_{i, x}(t)\right\|_{L^{1}} & =\mathcal{O}(1) \delta_{0}^{2}, \\
\left\|V_{i, x}(t)\right\|_{L^{\infty}},\left\|W_{i, x}(t)\right\|_{L^{\infty}} & =\mathcal{O}(1) \delta_{0}^{3},
\end{aligned}
$$

for all $t \in[\hat{t}, T]$.

Proof. Use the local invertibility of the map $\mathcal{G}(U ; \cdot, \cdot)$ and the estimates in Lemma 4.2. 
6. The Evolution of the COMponents.

This section investigates the evolution of the components $V_{i}$ and $W_{i}$ in the decomposition (5.10), which places a significant role in the proof of Lemma 3.1.

Lemma 6.1. The unique solution $(V, W)$ of decomposition (5.10) satisfies a $2 n \times 2 n$ system of balance laws with source of the form:

$$
\begin{array}{r}
\left(\begin{array}{c}
V \\
W
\end{array}\right)_{t}+\left[\left(\begin{array}{cc}
\Lambda & 0 \\
0 & \Lambda
\end{array}\right)\left(\begin{array}{c}
V \\
W
\end{array}\right)\right]_{x}-\left(\begin{array}{c}
V \\
W
\end{array}\right)_{x x}+\varepsilon\left(\begin{array}{ll}
B^{1} & B^{\sharp} \\
B^{b} & B^{2}
\end{array}\right)\left(\begin{array}{c}
V \\
W
\end{array}\right)=\varepsilon\left(\begin{array}{ll}
H^{1} & H^{\sharp} \\
H^{b} & H^{2}
\end{array}\right)\left(\begin{array}{c}
V_{0} \\
W_{0}
\end{array}\right) \\
-\varepsilon^{2} \int_{0}^{t}\left(\begin{array}{ll}
K^{1} & K^{\sharp} \\
K^{b} & K^{2}
\end{array}\right)\left(\begin{array}{c}
V(\tau) \\
W(\tau)
\end{array}\right) d \tau+\left(\begin{array}{c}
\Phi \\
\Psi
\end{array}\right),
\end{array}
$$

where $\Lambda$ is the $n \times n$ diagonal matrix with entries $\left\{\tilde{\lambda}_{i}\right\}$, the matrix $\mathcal{B} \doteq\left(\begin{array}{ll}B^{1} & B^{\sharp} \\ B^{b} & B^{2}\end{array}\right)$ is diagonally dominant, the matrix $\mathcal{H} \doteq\left(\begin{array}{cc}H^{1} & H^{\sharp} \\ H^{\mathrm{b}} & H^{2}\end{array}\right)$ is in $L^{1}\left(M_{2 n \times 2 n} ;[0,+\infty)\right)$ and the matrix $\mathcal{K} \doteq\left(\begin{array}{cc}K^{1} & K^{\sharp} \\ K^{b} & K^{2}\end{array}\right)$ is absolutely dominated by $\mathcal{B}$. More precisely,

$$
\begin{gathered}
\left\|\left(\begin{array}{ll}
B^{1} & B^{\sharp} \\
B^{b} & B^{2}
\end{array}\right)-\left(\begin{array}{cc}
\tilde{B} & 0 \\
0 & \tilde{B}
\end{array}\right)\right\|_{\infty}=\mathcal{O}\left(\left|U-U^{*}\right|,|V|,|W|\right)=\mathcal{O}(1) \delta_{0}, \\
\left\|\left(\begin{array}{cc}
H^{1} & H^{\sharp} \\
H^{b} & H^{2}
\end{array}\right)-\left(\begin{array}{cc}
\tilde{H} & 0 \\
0 & \tilde{H}
\end{array}\right)\right\|_{\infty}=\mathcal{O}\left(\left|U-U^{*}\right|,|V|,|W|\right)=\mathcal{O}(1) \delta_{0}, \\
\left\|\left(\begin{array}{cc}
K^{1} & K^{\sharp} \\
K^{b} & K^{2}
\end{array}\right)-\left(\begin{array}{cc}
\tilde{K} & 0 \\
0 & \tilde{K}
\end{array}\right)\right\|_{\infty}=\mathcal{O}\left(\left|U-U^{*}\right|,|V|,|W|\right)=\mathcal{O}(1) \delta_{0},
\end{gathered}
$$

and

$$
\begin{gathered}
B_{i i}^{1}-\sum_{j \neq i}\left|B_{j i}^{1}\right|-\sum_{j=1}^{n}\left|B_{j i}^{b}\right|>\beta+\mathcal{O}(1) \delta_{0}, \\
B_{i i}^{2}-\sum_{j \neq i}\left|B_{j i}^{2}\right|-\sum_{j=1}^{n}\left|B_{j i}^{\sharp}\right|>\beta+\mathcal{O}(1) \delta_{0}, \\
\varepsilon \int_{0}^{+\infty} \sum_{j=1}^{n}\left|K_{j i}^{1}(\varepsilon s)\right|+\sum_{j=1}^{n}\left|K_{j i}^{b}(\varepsilon s)\right| d s<\kappa+\mathcal{O}(1) \delta_{0}, \\
\varepsilon \int_{0}^{+\infty} \sum_{j=1}^{n}\left|K_{j i}^{2}(\varepsilon s)\right|+\sum_{j=1}^{n}\left|K_{j i}^{\sharp}(\varepsilon s)\right| d s<\kappa+\mathcal{O}(1) \delta_{0},
\end{gathered}
$$


where $\tilde{B}, \tilde{H}, \tilde{K}, \kappa$ and $\beta$ are given in Assumptions A. Furthermore, the component source terms $\Phi$ and $\Psi$ in (6.1) satisfy the bounds

$$
\begin{aligned}
\Phi_{i}, \Psi_{i} & =O(1) \cdot \sum_{j \neq i}\left(\left|V_{j} V_{i}\right|+\left|V_{j, x} V_{i}\right|+\left|V_{j} W_{i}\right|+\left|V_{j} W_{i, x}\right|+\left|W_{j} W_{i}\right|+\left|V_{j, x} W_{i}\right|\right. \\
& \left.+\left|V_{j, x} V_{i, x}\right|+\left|V_{j, x} W_{i, x}\right|+\left|W_{j} W_{i, x}\right|+\left|W_{j, x} W_{i, x}\right|\right) \longrightarrow \text { Transversal } \\
& +O(1) \cdot \sum_{j}\left|V_{j, x} W_{j}-V_{j} W_{j, x}\right| \longrightarrow \text { Change in speed, Linear } \\
& +O(1) \cdot \sum_{j}\left|V_{j}\left(\frac{W_{j}}{V_{j}}\right)_{x}\right|^{2} \cdot \chi_{\left\{\left|W_{j} / V_{j}\right|<3 \delta_{1}\right\}} \longrightarrow \text { Change in speed, Quadratic } \\
(6.9) \quad+ & O(1) \cdot \sum_{j}\left(\left|V_{j, x}\right|+\left|W_{j, x}\right|\right) \cdot\left|W_{j}-\theta_{j} V_{j}\right| . \longrightarrow \text { Cutoff }
\end{aligned}
$$

Proof. To begin with, upon differentiating (2.11) with respect to $x$ and $t$ respectively, one obtains the evolution equations of $U_{x}$ and $U_{t}$

$$
\begin{aligned}
& U_{x t}+\left(A(U) U_{x}\right)_{x}+\varepsilon(g(U))_{x}-\varepsilon H(\varepsilon t) U_{0, x}+\varepsilon^{2} \int_{0}^{t} K(\varepsilon(t-\tau)) U_{x}(\tau) d \tau-\left(U_{x}\right)_{x x}=0 \\
& U_{t t}+\left(A(U) U_{t}\right)_{x}+\varepsilon(\Delta(U, t, \varepsilon))_{t}-\left(U_{t}\right)_{x x}=\left(U_{x} \bullet A(U)\right) U_{t}-\left(U_{t} \bullet A(U)\right) U_{x} .
\end{aligned}
$$

Using the decomposition (5.10), we can rewrite the above equations as shown below. It should be noted that many of the calculations are omitted and we refer the reader to the appendix of [7] for more details. Here, we only choose to present the resulting equations after heavy computations.

$$
\begin{aligned}
\sum_{i}\left(V_{i, t}+\left(\tilde{\lambda}_{i} V_{i}\right)_{x}-V_{i, x x}\right)\left[\tilde{r}_{i}+V_{i} \tilde{r}_{i, V}+\theta_{i}^{\prime} \frac{W_{i}}{V_{i}} \tilde{r}_{i, \sigma}\right] & \\
+ & \sum_{i}\left(W_{i, t}+\left(\tilde{\lambda}_{i} W_{i}\right)_{x}-W_{i, x x}\right)\left[-\theta_{i}^{\prime} \tilde{r}_{i, \sigma}\right]+\varepsilon \sum_{i} V_{i}\left[D g(U) \tilde{r}_{i}-\tilde{r}_{i, U} g(U)\right]= \\
=\varepsilon \sum_{i}[ & \left.H(\varepsilon t) V_{0 i, x} \tilde{r}_{i}(0)-V_{i} \tilde{r}_{i, U} H(\varepsilon t) U_{0}\right] \\
- & -\varepsilon^{2}\left[\sum_{i} \int_{0}^{t} K(\varepsilon(t-\tau)) V_{i}(\tau) \tilde{r}_{i}(\tau) d \tau-V_{i} \tilde{r}_{i, U} \int_{0}^{t} K(\varepsilon(t-\tau)) U(\tau) d \tau\right] \\
& +\sum_{i} \alpha_{i},
\end{aligned}
$$


and

$$
\begin{aligned}
& \sum_{i}\left(V_{i, t}+\left(\tilde{\lambda}_{i} V_{i}\right)_{x}-V_{i, x x}\right)\left[W_{i} \tilde{r}_{i, V}+\theta_{i}^{\prime}\left(\frac{W_{i}}{V_{i}}\right)^{2} \tilde{r}_{i, \sigma}\right] \\
& +\sum_{i}\left(W_{i, t}+\left(\tilde{\lambda}_{i} W_{i}\right)_{x}-W_{i, x x}\right)\left[\tilde{r}_{i}-\theta_{i}^{\prime} \frac{W_{i}}{V_{i}} \tilde{r}_{i, \sigma}\right] \\
& -\sum_{i} \lambda_{i}^{*}\left\{\left(V_{i, t}+\left(\tilde{\lambda}_{i} V_{i}\right)_{x}-V_{i, x x}\right)\left[\tilde{r}_{i}+V_{i} \tilde{r}_{i, V}+\theta_{i}^{\prime} \frac{W_{i}}{V_{i}} \tilde{r}_{i, \sigma}\right]\right. \\
& \left.\quad-\left(W_{i, t}+\left(\tilde{\lambda}_{i} W_{i}\right)_{x}-W_{i, x x}\right)\left[\theta_{i}^{\prime} \tilde{r}_{i, \sigma}\right]\right\} \\
& +\varepsilon \sum_{i}\left(W_{i}-\lambda_{i}^{*} V_{i}\right)\left(D g(U) \tilde{r}_{i}-\tilde{r}_{i, U} g(U)\right) \\
& +\varepsilon \sum_{i} V_{i}\left(D g(U) A(U)-A(U) D g(U)-g(U) \bullet A(U)+V_{i} \tilde{r}_{i} \bullet D g(U)\right) \tilde{r}_{i} \\
& -\varepsilon \sum_{i}\left[\left(W_{0, i}-\lambda_{i}^{*} V_{0, i}\right) H(\varepsilon t) \tilde{r}_{i}(0)-\left(W_{i}-\lambda_{i}^{*} V_{i}\right) \tilde{r}_{i, U} H(\varepsilon t) U_{0}\right] \\
& -\varepsilon \sum_{i} V_{0, i}\left[H(\varepsilon t) A\left(U_{0}\right)-A(U) H(\varepsilon t)\right] \tilde{r}_{i}(0)+\varepsilon \sum_{i} V_{i}\left(H(\varepsilon t) U_{0} \bullet A(U)\right) \tilde{r}_{i} \\
& +\varepsilon^{2} \sum_{i}\left[\int_{0}^{t} K(\varepsilon(t-\tau))\left(W_{i}(\tau)-\lambda_{i}^{*} V_{i}(\tau)\right) \tilde{r}_{i}(\tau) d \tau\right. \\
& +\varepsilon^{2} \sum_{i}\left[\int_{0}^{t} V_{i}(\tau) K(\varepsilon(t-\tau)) A(U(\tau)) \tilde{r}_{i}(\tau) d \tau-A(U) \int_{0}^{t} V_{i}(\tau) K(\varepsilon(t-\tau)) \tilde{r}_{i}(\tau) d \tau\right]
\end{aligned}
$$

$$
-\varepsilon^{2} \sum_{i} V_{i}\left(\int_{0}^{t} K(\varepsilon(t-\tau)) U(\tau) d \tau \bullet A(U)\right) \tilde{r}_{i}=\sum_{i} \beta_{i}-\lambda_{i}^{*} \alpha_{i},
$$

where $\alpha_{i}$ and $\beta_{i}$ are as given in Appendix A of [7]. Observe that system (6.11)-(6.12) can be written in the form:

$$
\begin{aligned}
\left(\frac{\partial \mathcal{G}}{\partial(V, W)}\right)\left(\begin{array}{c}
V_{t}+(\tilde{\Lambda} V)_{x}-V_{x x} \\
W_{t}+(\tilde{\Lambda} W)_{x}-W_{x x}
\end{array}\right)+\varepsilon \bar{B}\left(\begin{array}{c}
V \\
W
\end{array}\right)= & \varepsilon \bar{H}\left(\begin{array}{c}
V_{0} \\
W_{0}
\end{array}\right)-\varepsilon^{2} \int_{0}^{t} \bar{K}\left(\begin{array}{c}
V(\tau) \\
W(\tau)
\end{array}\right) d \tau \\
& +\left(\begin{array}{c}
\sum_{i} \alpha_{i} \\
\sum_{i} \beta_{i}-\lambda_{i}^{*} \alpha_{i}
\end{array}\right)
\end{aligned}
$$

Here, $\bar{B}, \bar{H}$ and $\bar{K}$ are $2 \times(2 n)$ matrices with entries:

$$
\begin{gathered}
\bar{B} \doteq\left(\begin{array}{cccccc}
\bar{B}_{11} & \ldots & \bar{B}_{1 n} & 0 & \ldots & 0 \\
\bar{B}_{21} & \ldots & \bar{B}_{2 n} & \bar{B}_{2(n+1)} & \ldots & \bar{B}_{2(2 n)}
\end{array}\right), \\
\bar{B}_{1 j}=D g(u) \tilde{r}_{j}-\tilde{r}_{j, U} g(U)+\tilde{r}_{j, U} H(\varepsilon t) U_{0}-\varepsilon \tilde{r}_{j, U} \int_{0}^{t} K(\varepsilon(t-\tau)) U(\tau) d \tau,
\end{gathered}
$$

for $j=1, \ldots, n$,

$$
\bar{B}_{1 j}=0 \quad j=n, \ldots, 2 n,
$$




$$
\begin{aligned}
\bar{B}_{2 j} & =\left[D g(u) A(u)-A(u) D g(u)-g(u) \bullet A(u)+v_{j}\left(\tilde{r}_{j} \bullet D g(u)\right)\right] \tilde{r}_{j} \\
& -\lambda_{j}^{*}\left[D g(U) \tilde{r}_{j}-\tilde{r}_{j, U} g(U)\right]+\left(H(\varepsilon t) U_{0} \bullet A(U)\right) \tilde{r}_{j}-\lambda_{j}^{*} \tilde{r}_{j, U} H(\varepsilon t) U_{0} \\
& -\varepsilon\left(\int_{0}^{t} K(\varepsilon(t-\tau)) U(\tau) d \tau \bullet A(U)\right) \tilde{r}_{j}+\varepsilon \lambda_{j}^{*} \tilde{r}_{j, U} \int_{0}^{t} K(\varepsilon(t-\tau)) U(\tau) d \tau
\end{aligned}
$$

for $j=1, \ldots, n$,

$$
\bar{B}_{2 j}=D g(u) \tilde{r}_{j}-\tilde{r}_{j, U} g(U)+\tilde{r}_{j, U} H(\varepsilon t) U_{0}-\varepsilon \tilde{r}_{j, U} \int_{0}^{t} K(\varepsilon(t-\tau)) U(\tau) d \tau,
$$

for $j=n, \ldots, 2 n$;

$$
\begin{aligned}
& \bar{H} \doteq\left(\begin{array}{cccccc}
H(\varepsilon t) \tilde{r}_{1}(0) & \ldots & H(\varepsilon t) \tilde{r}_{n}(0) & 0 & \ldots & 0 \\
\bar{H}_{21} & \ldots & \bar{H}_{2 n} & H(\varepsilon t) \tilde{r}_{1}(0) & \ldots & H(\varepsilon t) \tilde{r}_{n}(0)
\end{array}\right), \\
& \bar{H}_{2 j}=-\lambda_{j}^{*} H(\varepsilon t) \tilde{r}_{j}(0)+\left[H(\varepsilon t) A\left(U_{0}\right)-A(U) H(\varepsilon t)\right] \tilde{r}_{j}(0) ; \\
& \bar{K} \doteq\left(\begin{array}{cccccc}
\bar{K}_{11} & \ldots & \bar{K}_{1 n} & 0 & \ldots & 0 \\
\bar{K}_{21} & \ldots & \bar{K}_{2 n} & \bar{K}_{11} & \ldots & \bar{K}_{1 n}
\end{array}\right) \text {, } \\
& \bar{K}_{1 j}=K(\varepsilon(t-\tau)) \tilde{r}_{j}(\tau) \\
& \bar{K}_{2 j}=-\lambda_{j}^{*} K(\varepsilon(t-\tau)) \tilde{r}_{j}(\tau)+[K(\varepsilon(t-\tau)) A(U(\tau))-A(U) K(\varepsilon(t-\tau))] \tilde{r}_{j}(\tau),
\end{aligned}
$$

for $j=1, \ldots, n$.

In Lemma 5.1, it is shown that

$$
\frac{\partial \mathcal{G}_{i}}{\partial\left(V_{i}, W_{i}\right)} \rightarrow\left(\begin{array}{cc}
\tilde{r}_{i} & 0 \\
-\lambda_{i}^{*} \tilde{r}_{i} & \tilde{r}_{i}
\end{array}\right), \quad \quad \text { as }\left(V_{i}, W_{i}\right) \rightarrow(0,0)
$$

and it is therefore invertible in a neighborhood of $\left(U^{*}, 0,0\right)$. Hence, by $(6.13)$, we obtain the evolution equation (6.1). By employing the properties of $\frac{\partial \mathcal{G}}{\partial(V, W)}$ already derived in Lemma 5.1, Assumptions A and estimates (5.6), we verify the statements (6.2)-(6.4), which imply (6.5)-(6.8). To complete the proof it suffices to investigate the various component source terms $\Phi=\mathcal{O}(1) \sum_{i} \alpha_{i}$ and $\Psi=\mathcal{O}(1) \sum_{i} \beta_{i}-\lambda_{i}^{*} \alpha_{i}$ that appear in the force of (6.1), where $\alpha_{i}$ and $\beta_{i}$ are given explicitly in [7]. One can follow similar calculations to those presented in Appendix A of [7] to verify that the source $(\Phi, \Psi)$ can be written in the given form (6.9). Here, we choose to omit this part of the proof.

In view of the above lemma, the component source terms $\Phi, \Psi$ belong to the four categories given in (6.9), as in [3, 7]. The name that each category is labeled comes from the construction of the decomposition and they are the same as in [7]. Having the above result, we prove Lemma 3.1 in the following 4 subsections. For convenience, we adopt the terminology introduced in [7]:

Definition 6.2. We call a scalar function $\xi=\xi(t, x)$ controllable if (3.6) implies

$$
\int_{\hat{t}}^{T} \int|\xi(t, x)| d x d t=\mathcal{O}(1) \delta_{0}^{2}
$$

Thus Lemma 3.1 amounts to showing that $\left|\Phi_{i}(t, x)\right|$ and $\left|\Psi_{i}(t, x)\right|$ are controllable for all $i=1, \ldots, n$. We employ an interaction potential to control the transversal terms in Section 6.1. The terms that belong to the change in speed category whether linear or quadratic, are handled by means of the area and length functionals of suitable planar curves introduced in Sections 6.2-6.3. Last, in Section 6.4, by 
employing energy methods, it is shown that the cutoff terms are monitored by terms in the preceding categories and are therefore controllable.

The estimates of the wave interactions follow closely that ones in [7], which are certainly a generalization of the fundamental techniques first introduced in [3]. In this paper, we would like to emphasize the structure of system (6.1) and most importantly to indicate the role of the $\varepsilon$-terms of (6.1) in the proof and the treatment of the memory term.

For future use, we introduce the following constants:

$$
M_{B}=\|\mathcal{B}\|_{\infty}, \quad M_{H}=\varepsilon \int_{0}^{+\infty} \sup _{x}|\mathcal{H}| d t, \quad M_{K}=\varepsilon \int_{0}^{+\infty} \sup _{x}|\mathcal{K}| d t .
$$

The presence of the $\varepsilon$ factor is due to the rescaled coordinates. Indeed, notice that $M_{B}, M_{H}$ and $M_{K}$ are almost equal to the corresponding constants if you replace $\mathcal{B}, \mathcal{H}$ and $\mathcal{K}$ by $\tilde{B}, \tilde{H}$ and $\tilde{K}$ respectively, (6.2)-(6.4) imply that

$M_{B}=\|\tilde{B}\|_{\infty}+\mathcal{O}(1) \delta_{0}, \quad M_{H}=\int_{0}^{+\infty}|\tilde{H}| d t+\mathcal{O}(1) \delta_{0}, \quad M_{K}=\int_{0}^{+\infty}|\tilde{K}| d t+\mathcal{O}(1) \delta_{0}$

are independent of $\varepsilon$.

6.1. Transversal terms. In this subsection, we prove that the transversal terms are controllable, i.e. if the assumptions of Lemma 3.1 hold, then we establish the following estimate:

$$
\begin{gathered}
\int_{\hat{t}}^{T} \int \sum_{j \neq k}\left(\left|V_{j} V_{k}\right|+\left|V_{j, x} V_{k}\right|+\left|V_{j} W_{k}\right|+\left|V_{j} W_{k, x}\right|+\left|W_{j} W_{k}\right|+\left|W_{j} W_{k, x}\right|\right. \\
\left.+\left|V_{j, x} V_{k, x}\right|+\left|V_{j, x} W_{k}\right|+\left|V_{j, x} W_{k, x}\right|+\left|W_{j, x} W_{k, x}\right|\right) d x d t=O(1) \delta_{0}^{2},
\end{gathered}
$$

for sufficiently small $\delta_{0}$. By the strict hyperbolicity of system (6.1), there exists a positive constant $c>0$ such that

$$
\inf _{t, x} \tilde{\lambda}_{j}-\sup _{t, x} \tilde{\lambda}_{i} \geq c>0, \quad \forall i<j .
$$

First, we prove the result for only those terms that do not involve derivatives.

Lemma 6.3. Let $(V, W)$ be solution of $(6.1)$ defined for $t \in[0, T]$. Then for $i \neq j$,

$$
\int_{0}^{T} \int\left|V_{i} V_{j}\right|+\left|V_{i} W_{j}\right|+\left|W_{i} W_{j}\right| d x d t=\frac{\mathcal{O}(1)}{c} \delta_{0}^{2} .
$$

Proof. Consider the interaction potential functional

$$
\mathcal{Q}(z, \tilde{z})=\iint \mathcal{K}(x-y) z(x) \tilde{z}(y) d x d y,
$$

where the kernel $\mathcal{K}$ is given by

$$
\mathcal{K}(s)= \begin{cases}\frac{1}{c} & s \geq 0 \\ \frac{1}{c} e^{c s / 2} & s<0 .\end{cases}
$$


To verify (6.22), apply the functional $\mathcal{Q}$ on the pairs $\left(V_{i}, V_{j}\right),\left(V_{i}, W_{j}\right),\left(W_{i}, W_{j}\right)$ for $i<j$. If system (6.1) is homogeneous, then by direct calculations, we get

$$
\begin{aligned}
& \frac{d}{d t} \mathcal{Q}\left(V_{i}(t), V_{j}(t)\right) \leq-\int\left|V_{i}(x)\right|\left|V_{j}(x)\right| d x \\
& \quad-\varepsilon \iint \mathcal{K}(x-y)\left[\operatorname { s g n } V _ { i } ( x ) \sum _ { k } \left(B_{i k}^{1} V_{k}(x)+B_{i k}^{\sharp} W_{k}(x)\right.\right. \\
& \left.\quad-H_{i k}^{1} V_{0, k}(x)-H_{i k}^{\sharp} W_{0, k}(x)+\varepsilon \int_{0}^{t} K_{i k}^{1} V_{k}(\tau, x)+K_{i k}^{\sharp} W_{k}(\tau, x) d \tau\right)\left|V_{j}(y)\right| \\
& \quad+\left|V_{i}(x)\right| \operatorname{sgn} V_{j}(y) \sum_{k}\left(B_{j k}^{1} V_{k}(y)+B_{j k}^{\sharp} W_{k}(y)-H_{j k}^{1} V_{0, k}(y)-H_{j k}^{\sharp} W_{0, k}(y)\right. \\
& \left.\left.\left.\quad+\varepsilon \int_{0}^{t} K_{j k}^{1} V_{k}(\tau, y)+K_{j k}^{\sharp} W_{k}(\tau, y) d \tau\right)\right)\right] d y d x
\end{aligned}
$$

since $c \mathcal{K}^{\prime}-2 \mathcal{K}^{\prime \prime}$ is the delta function centered at the origin. Let $M_{B}, M_{H}$ and $M_{K}$ as given in (6.18). Recalling (5.18), (6.18) and the integrability in time of the component terms $\varepsilon\left\|V_{k}\right\|_{L^{1}}$, we obtain

$$
\begin{aligned}
& \int_{\hat{t}}^{T} \int\left|V_{i}(x) V_{j}(x)\right| d x d t \leq \mathcal{Q}\left(V_{i}(\hat{t}), V_{j}(\hat{t})\right) \\
& \quad+\frac{\varepsilon}{c} M_{B} \sum_{k} \int_{\hat{t}}^{T}\left[\left(\left\|V_{k}(t)\right\|_{L^{1}}+\left\|W_{k}(t)\right\|_{L^{1}}\right)\left\|V_{j}\right\|_{L^{1}}+\left\|V_{i}\right\|_{L^{1}}\left(\left\|\left.V_{k}(t)\right|_{L^{1}}+\right\| W_{k}(t) \|_{L^{1}}\right)\right] d t \\
& \quad+\frac{\varepsilon}{c} \sum_{k} \int_{\hat{t}}^{T}\left[\left(\left\|V_{0}\right\|_{L^{1}} \sup _{x}\left|H_{i k}^{1}\right|+\left\|W_{0}\right\|_{L^{1}} \sup _{x}\left|H_{i k}^{\sharp}\right|\right)\left\|V_{j}\right\|_{L^{1}}\right. \\
& \left.\quad+\left\|V_{i}\right\|_{L^{1}}\left(\left\|V_{0}\right\|_{L^{1}} \sup _{x}\left|H_{j k}^{1}\right|+\left\|W_{0}\right\|_{L^{1}} \sup _{x}\left|H_{j k}^{\sharp}\right|\right)\right] d t \\
& \quad+\mathcal{O}(1) \frac{\varepsilon}{c} M_{K} \delta_{0} \int_{\hat{t}}^{T}\left\|V_{j}(t)\right\|_{L^{1}}+\left\|V_{i}(t)\right\|_{L^{1}} d t \\
& \leq \\
& \frac{1}{c}\left\|V_{i}(\hat{t})\right\|_{L^{1}}\left\|V_{j}(\hat{t})\right\|_{L^{1}}+\frac{\varepsilon}{c} \delta_{0}^{2} \int_{\hat{t}}^{T} \sup _{x}|\mathcal{H}| d t+\mathcal{O}(1) \frac{\varepsilon}{c} \delta_{0}\left(M_{B}+M_{K}\right) \int_{\hat{t}}^{T} \sum_{k}\left\|V_{k}\right\|_{L^{1}} d t
\end{aligned}
$$

$$
=\frac{1}{c}\left\|V_{i}(\hat{t})\right\|_{L^{1}}\left\|V_{j}(\hat{t})\right\|_{L^{1}}+\frac{\mathcal{O}(1)}{c} \delta_{0}^{2}
$$

which easily reduces to (6.22). Similarly, we treat the terms $V_{i} W_{j}, W_{i} W_{j}$. Hence, the result clearly holds for the homogeneous system. To complete the proof, consider the fundamental solution $(\Gamma, \tilde{\Gamma})$ to system $(6.1)$. In view of the above analysis, the fundamental solution $(\Gamma, \tilde{\Gamma})$ satisfy $(6.22)$. Consequently, it suffices to write the solution $(V, W)$ of $(6.1)$ in terms of $(\Gamma, \tilde{\Gamma})$ and use the corresponding estimates (6.22) on the pairs $\left(\Gamma_{i}, \Gamma_{j}\right),\left(\Gamma_{i}, \tilde{\Gamma}_{j}\right)$ and $\left(\tilde{\Gamma}_{i}, \tilde{\Gamma}_{j}\right)$. The result follows.

The next step would be to establish the estimate (6.20) for terms of the type $\left|V_{j, x} V_{k}\right|,\left|V_{j} W_{k, x}\right|,\left|V_{j, x} W_{k}\right|$ and $\left|W_{j} W_{k, x}\right|$. We can express $V_{j, x}$ by employing the heat kernel. Then, combining the $L^{1}$ estimates in Lemma 5.2, we deduce that the integral

$$
\int_{\hat{t}}^{T} \int\left|V_{j, x} V_{k}\right| d x d t
$$


is monitored by the integral (6.22), that is already estimated and this provides the desired bound. This procedure can also be similarly extended to the remaining transversal terms, $\left|V_{i, x} W_{j, x}\right|,\left|V_{i, x} W_{j, x}\right|$ and $\left|W_{i, x} W_{j, x}\right|$ by employing the terms that are already treated. For more details see $[3,7]$. Thus we have shown that all transversal terms are controllable and (6.20) is valid.

6.2. Swept Area functional. The aim here is to study the "change in speed, linear" terms and prove

$$
\int_{\hat{t}}^{T} \int\left|W_{i, x} V_{i}-W_{i} V_{i, x}\right| d x d t=\mathcal{O}(1) \delta_{0}^{2}
$$

assuming that (3.5) and (3.6) hold. As it was first demonstrated in the fundamental work of Bianchini and Bressan $[3,1,2]$, we should introduce the planar curves

$$
\gamma_{i}(t, x)=\left(\int_{-\infty}^{x} V_{i}(t, y) d y, \int_{-\infty}^{x} W_{i}(t, y) d y\right)
$$

and investigate their evolution in time. Because of the coupling of the system (6.1), we further need to study the $\tilde{\gamma}_{i}$ curves given by

$$
\tilde{\gamma}_{i}(t, x)=\left(\int_{-\infty}^{x} W_{i}(t, y) d y, \int_{-\infty}^{x} V_{i}(t, y) d y\right) .
$$

The motivation to employ these additional curves $\tilde{\gamma}_{i}$ has already been illustrated by Christoforou in [7]. By virtue of system (6.1), these curves $\gamma_{i}$ and $\tilde{\gamma}_{i}$ evolve in time according to the following equations:

$$
\begin{aligned}
\gamma_{i, t} & +\tilde{\lambda}_{i} \gamma_{i, x}-\gamma_{i, x x}+\varepsilon \sum_{k=1}^{n}\left(\int_{-\infty}^{x} B_{i k}^{1} V_{k}+B_{i k}^{\sharp} W_{k} d y, \int_{-\infty}^{x} B_{i k}^{b} V_{k}+B_{i k}^{2} W_{k} d y\right) \\
& =\varepsilon \sum_{k=1}^{n}\left(\int_{-\infty}^{x} H_{i k}^{1} V_{0, k}+H_{i k}^{\sharp} W_{0, k} d y, \int_{-\infty}^{x} H_{i k}^{b} V_{0, k}+H_{i k}^{2} W_{0, k} d y\right) \\
- & \varepsilon^{2} \sum_{k=1}^{n} \int_{0}^{t}\left(\int_{-\infty}^{x} K_{i k}^{1} V_{k}(\tau)+K_{i k}^{\sharp} W_{k}(\tau) d y, \int_{-\infty}^{x} K_{i k}^{b} V_{k}(\tau)+K_{i k}^{2} W_{k}(\tau) d y\right) d \tau
\end{aligned}
$$

$$
+\left(\int_{-\infty}^{x} \Phi_{i}(t, y) d y, \int_{-\infty}^{x} \Psi_{i}(t, y) d y\right)
$$$$
\tilde{\gamma}_{i, t}+\tilde{\lambda}_{i} \tilde{\gamma}_{i, x}-\tilde{\gamma}_{i, x x}+\varepsilon \sum_{k=1}^{n}\left(\int_{-\infty}^{x} B_{i k}^{b} V_{k}+B_{i k}^{2} W_{k} d y, \int_{-\infty}^{x} B_{i k}^{1} V_{k}+B_{i k}^{\sharp} W_{k} d y\right)
$$$$
=\varepsilon \sum_{k=1}^{n}\left(\int_{-\infty}^{x} H_{i k}^{b} V_{0, k}+H_{i k}^{2} W_{0, k} d y, \int_{-\infty}^{x} H_{i k}^{1} V_{0, k}+H_{i k}^{\sharp} W_{0, k} d y\right)
$$$$
-\varepsilon^{2} \sum_{k=1}^{n} \int_{0}^{t}\left(\int_{-\infty}^{x} K_{i k}^{b} V_{k}(\tau)+K_{i k}^{2} W_{k}(\tau) d y, \int_{-\infty}^{x} K_{i k}^{1} V_{k}(\tau)+K_{i k}^{\sharp} W_{k}(\tau) d y\right) d \tau
$$

$$
+\left(\int_{-\infty}^{x} \Psi_{i}(t, y) d y, \int_{-\infty}^{x} \Phi_{i}(t, y) d y\right) .
$$


Now, we consider the area functional $\mathcal{A}$

$$
\mathcal{A}(\gamma, \zeta)=\frac{1}{2} \iint_{x<y}\left|\gamma_{x}(x) \wedge \zeta_{x}(y)\right| d x d y
$$

associated with an ordered pair of curves $(\gamma, \zeta)$ as defined in [7]. It should be noted that this is a generalization of the definition in $[3,1,2]$. In order to derive $(6.26)$, we employ the functionals $\mathcal{A}\left(\gamma_{i}, \gamma_{j}\right)$ and $\mathcal{A}\left(\gamma_{i}, \tilde{\gamma}_{j}\right)$ for $i, j=1, \ldots, n$. It should be noted that if $\gamma_{i}$ is a closed curve, $\mathcal{A}\left(\gamma_{i}, \gamma_{i}\right)$ provides an upper bound for the sum of the areas of the regions enclosed by the curve counting the corresponding winding number. It will be shown that the area functionals are monotonically decreasing, because of the presence of the memory term, which appears as the $\varepsilon$-terms in system (6.29)-(6.30). This is the result of the next lemma.

Lemma 6.4. Let $(V, W)$ be solution to (6.1) for $t \in[0, T]$ and assume that the maps $x \mapsto V(t, x), x \mapsto W(t, x)$ and $x \mapsto \tilde{\lambda}(t, x)$ are $\mathcal{C}^{1,1}$. Then the area functionals satisfy:

$$
\begin{aligned}
\sum_{i, j} \frac{d}{d t} & {\left[\mathcal{A}\left(\gamma_{i}(t), \gamma_{j}(t)\right)+\mathcal{A}\left(\gamma_{i}(t), \tilde{\gamma}_{j}(t)\right)\right] \leq-\sum_{i}\left\|W_{i, x} V_{i}-W_{i} V_{i, x}\right\| } \\
& -\varepsilon\left(\beta+\mathcal{O}(1) \delta_{0}\right) \sum_{i, j}\left[\mathcal{A}\left(\gamma_{i}(t), \gamma_{j}(t)\right)+\mathcal{A}\left(\gamma_{i}(t), \tilde{\gamma}_{j}(t)\right)\right]+\varepsilon \Omega(t) \\
& +\sum_{i, j}\left(\left\|V_{i}(t)\right\|_{L^{1}}+\left\|W_{i}\right\|_{L^{1}}\right)\left(\left\|\Phi_{j}\right\|_{L^{1}}+\left\|\Psi_{j}(t)\right\|_{L^{1}}\right)
\end{aligned}
$$

where the function $\Omega$ satisfies

(6.33)

$\varepsilon \int_{\hat{t}}^{T} \Omega(t) d t=\varepsilon\left(\kappa+\mathcal{O}(1) \delta_{0}\right) \sum_{i, j} \int_{\hat{t}}^{T}\left[\mathcal{A}\left(\gamma_{i}(t), \gamma_{j}(t)\right)+\mathcal{A}\left(\gamma_{i}(t), \tilde{\gamma}_{j}(t)\right)\right] d t+\mathcal{O}(1) \delta_{0}^{2}$.

Proof. At each $x$ where $\gamma_{i, x} \neq 0$, define the vector $\eta_{i}(x)$ in $\mathbb{R}^{2}$ to be

$$
\gamma_{i, x}(x) \wedge \vec{v}=\left|\gamma_{i, x}\right|\left\langle\eta_{i}(x), \vec{v}\right\rangle, \quad \text { i.e. } \quad \eta_{i}(x)=\left(-\frac{W_{i}(x)}{\left|\gamma_{i, x}\right|}, \frac{V_{i}(x)}{\left|\gamma_{i, x}\right|}\right) .
$$

For fixed $x$, consider the projection of $\gamma_{j}$ along the vector $\eta_{i}(x)$,

$$
y \mapsto \chi^{\eta_{i}, \gamma_{j}}(y)=\left\langle\eta_{i}(x), \gamma_{j}(y)\right\rangle
$$

We quote from [7] that

$$
\begin{aligned}
\frac{d}{d t}\left(\mathcal{A}\left(\gamma_{i}, \gamma_{j}\right)+\mathcal{A}\left(\gamma_{j}, \gamma_{i}\right)\right)= & \frac{1}{2} \int\left|\gamma_{i, x}(x)\right| \frac{d}{d t}\left(T V_{y} \chi^{\eta_{i}(x), \gamma_{j}}\right) d x \\
& +\frac{1}{2} \int\left|\gamma_{j, x}(x)\right| \frac{d}{d t}\left(T V_{y} \chi^{\eta_{j}(x), \gamma_{i}}\right) d x
\end{aligned}
$$

and

$$
\frac{d}{d t}\left(T V_{y} \chi^{\eta_{i}, \gamma_{j}}\right)=-\operatorname{sgn}\left\langle\eta_{i}(x), \gamma_{j, x x}(x)\right\rangle 2 \sum_{\alpha}(-1)^{\alpha}\left\langle\eta_{i}(x), \gamma_{j, t}\left(y_{\alpha}^{i, j}\right)\right\rangle
$$

where $y_{\alpha}^{i, j}$ denote the finite number of points at which the projection function $\chi^{\eta_{i}(x), \gamma_{j}}(y)$ changes monotonicity, such that $y_{0}^{i, i}=x$. See Figure 1. For more details on this construction, see pp. 24-26 in [7]. 


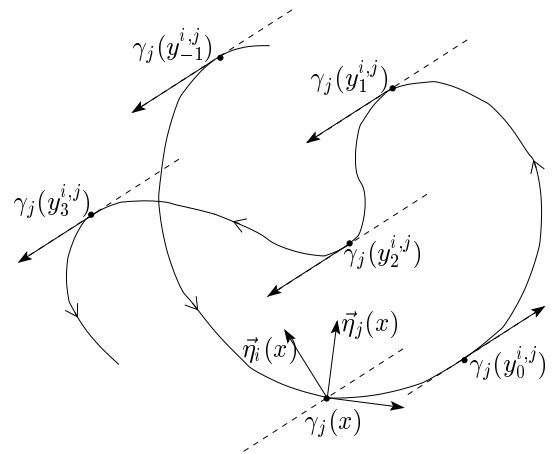

(a) The curve $\gamma_{j}(y)$ and the vector $\eta_{i}(x)$ perpendicular to $\gamma_{j, x}\left(y_{\alpha}^{i, j}\right)$.

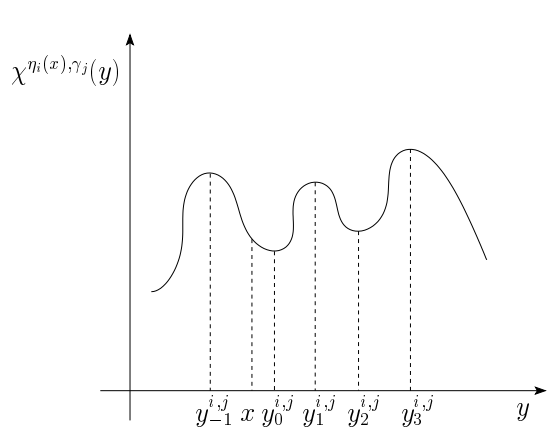

(b) Graph of $\chi^{\eta_{i}(x), \gamma_{j}}(y)$.

FIGURE 1.

Substituting in (6.36) and using further identities of $\eta_{i}$ and $\chi^{\eta_{i}(x), \gamma_{j}}$, we arrive at

$$
\begin{aligned}
& \frac{d}{d t}\left(\mathcal{A}\left(\gamma_{i}, \gamma_{j}\right)+\mathcal{A}\left(\gamma_{j}, \gamma_{i}\right)\right) \leq \\
& \leq-\int\left|\gamma_{i, x}(x) \wedge \gamma_{j, x x}\left(y_{0}^{i, j}\right)\right| d x+\left\|V_{i}(t)\right\|_{L^{1}}\left\|\Psi_{j}(t)\right\|_{L^{1}}+\left\|W_{i}(t)\right\|_{L^{1}}\left\|\Phi_{j}(t)\right\|_{L^{1}} \\
& -\frac{\varepsilon}{2} \iint\left|\gamma_{i, x}(x)\right| \operatorname{sgn}\left\langle\eta_{i}(x), \gamma_{j, x}(y)\right\rangle\left\langle\eta_{i}(x), b_{j}(y)\right\rangle d y d x \\
& +\frac{\varepsilon}{2} \iint\left|\gamma_{i, x}(x)\right| \operatorname{sgn}\left\langle\eta_{i}(x), \gamma_{j, x}(y)\right\rangle\left\langle\eta_{i}(x), h_{j}(y)\right\rangle d y d x \\
& -\frac{\varepsilon^{2}}{2} \iint\left|\gamma_{i, x}(x)\right| \operatorname{sgn}\left\langle\eta_{i}(x), \gamma_{j, x}(y)\right\rangle\left\langle\eta_{i}(x), k_{j}(y)\right\rangle d y d x \\
& -\int\left|\gamma_{j, x}(x) \wedge \gamma_{i, x x}\left(y_{0}^{j, i}\right)\right| d x+\left\|V_{j}(t)\right\|_{L^{1}}\left\|\Psi_{i}(t)\right\|_{L^{1}}+\left\|W_{j}(t)\right\|_{L^{1}}\left\|\Phi_{i}(t)\right\|_{L^{1}} \\
& -\frac{\varepsilon}{2} \iint\left|\gamma_{j, x}(x)\right| \operatorname{sgn}\left\langle\eta_{j}(x), \gamma_{i, x}(y)\right\rangle\left\langle\eta_{j}(x), b_{i}(y)\right\rangle d y d x \\
& +\frac{\varepsilon}{2} \iint\left|\gamma_{j, x}(x)\right| \operatorname{sgn}\left\langle\eta_{j}(x), \gamma_{i, x}(y)\right\rangle\left\langle\eta_{j}(x), h_{i}(y)\right\rangle d y d x
\end{aligned}
$$

$$
-\frac{\varepsilon^{2}}{2} \iint\left|\gamma_{j, x}(x)\right| \operatorname{sgn}\left\langle\eta_{j}(x), \gamma_{i, x}(y)\right\rangle\left\langle\eta_{j}(x), k_{i}(y)\right\rangle d y d x,
$$

where

$$
\begin{gathered}
b_{l}(y)=\left(\sum_{k} B_{l k}^{1} V_{k}(y)+B_{l k}^{\sharp} W_{k}(y), \sum_{k} B_{l k}^{b} V_{k}(y)+B_{l k}^{2} W_{k}(y)\right), \\
h_{l}(y)=\left(\sum_{k} H_{l k}^{1} V_{0, k}(y)+H_{l k}^{\sharp} W_{0, k}(y), \sum_{k} H_{l k}^{b} V_{0, k}(y)+H_{l k}^{2} W_{0, k}(y)\right),
\end{gathered}
$$


and

$$
k_{l}(y)=\left(\sum_{k} \int_{0}^{t} K_{l k}^{1} V_{k}(\tau, y)+K_{l k}^{\sharp} W_{k}(\tau, y) d \tau, \sum_{k} \int_{0}^{t} K_{l k}^{b} V_{k}(\tau, y)+K_{l k}^{2} W_{k}(\tau, y) d \tau\right) .
$$

Similarly, we estimate

$$
\frac{d}{d t}\left(\mathcal{A}\left(\gamma_{i}, \tilde{\gamma}_{j}\right)+\mathcal{A}\left(\gamma_{j}, \tilde{\gamma}_{i}\right)\right)
$$

Summing (6.38) and (6.39) over all $i, j=1, \ldots, n$, yields

$$
\begin{aligned}
\frac{d}{d t} \sum_{i, j}\left(\mathcal{A}\left(\gamma_{i}, \gamma_{j}\right)+\right. & \left.\mathcal{A}\left(\gamma_{i}, \tilde{\gamma}_{j}\right)\right) \leq-\sum_{i} \int\left|\gamma_{i, x}(x) \wedge \gamma_{i, x x}(x)\right| d x+b(t, \varepsilon)+h(t, \varepsilon)+k(t, \varepsilon) \\
& +\sum_{i, j}\left(\left\|V_{i}(t)\right\|_{L^{1}}+\left\|W_{i}\right\|_{L^{1}}\right)\left(\left\|\Phi_{j}(t)\right\|_{L^{1}}+\left\|\Psi_{j}(t)\right\|_{L^{1}}\right)
\end{aligned}
$$

where

$$
\begin{aligned}
b(t ; \varepsilon) \doteq \sum_{i, j=1, n}\{- & \frac{\varepsilon}{2} \iint\left\{B_{j j}^{1}-\sum_{k \neq j}\left|B_{k j}^{1}\right|-\sum_{k}\left|B_{k j}^{b}\right|\right\}\left|V_{i}(x) W_{j}(y)-W_{i}(x) V_{j}(y)\right| d y d x \\
& -\frac{\varepsilon}{2} \iint\left\{B_{j j}^{1}-\sum_{k \neq j}\left|B_{k j}^{1}\right|-\sum_{k}\left|B_{k j}^{b}\right|\right\}\left|V_{i}(x) V_{j}(y)-W_{i}(x) W_{j}(y)\right| d y d x \\
& \left.+\varepsilon \mathcal{O}(1) \delta_{0}\left(\left\|V_{j}\right\|_{L^{1}}\left\|W_{i}\right\|_{L^{1}}+\left\|V_{j}\right\|_{L^{1}}\left\|V_{i}\right\|_{L^{1}}+\left\|W_{j}\right\|_{L^{1}}\left\|W_{i}\right\|_{L^{1}}\right)\right\} \\
& \leq-\varepsilon(\beta-\mu) \sum_{i, j}\left(\mathcal{A}\left(\gamma_{i}, \gamma_{j}\right)+\mathcal{A}\left(\gamma_{i}, \tilde{\gamma}_{j}\right)\right)+\varepsilon \mathcal{O}(1) \delta_{0}^{2}(\|V\|+\|W\|),
\end{aligned}
$$

$$
\begin{aligned}
h(t, \varepsilon) \doteq & \frac{\varepsilon}{2} \sum_{i, j} \iint \sum_{k}\left|\left\langle\left(-W_{i}(x), V_{i}(x)\right),\left(H_{j k}^{1} V_{k}(y), H_{j k}^{2} W_{k}(y)\right)\right\rangle\right| \\
& +\sum_{k}\left|\left\langle\left(-W_{i}(x), V_{i}(x)\right),\left(H_{j k}^{\sharp} W_{k}(y), H_{j k}^{b} V_{k}(y)\right)\right\rangle\right| \\
& +\sum_{k}\left|\left\langle\left(-W_{i}(x), V_{i}(x)\right),\left(H_{j k}^{2} W_{k}(y), H_{j k}^{1} V_{k}(y)\right)\right\rangle\right| \\
& +\sum_{k}\left|\left\langle\left(-W_{i}(x), V_{i}(x)\right),\left(H_{j k}^{b} V_{k}(y), H_{j k}^{\sharp} W_{k}(y)\right)\right\rangle\right| d x d y \\
\leq & \varepsilon \sup _{x}|\mathcal{H}| \mathcal{O}(1) \sum_{i, j}\left(\left\|V_{i}\right\|_{L^{1}}\left\|V_{j}\right\|_{L^{1}}+\left\|V_{i}\right\|_{L^{1}}\left\|W_{j}\right\|_{L^{1}}+\left\|W_{i}\right\|_{L^{1}}\left\|W_{j}\right\|_{L^{1}}\right)
\end{aligned}
$$

$(6.41)=\mathcal{O}(1) \delta_{0}^{2} \varepsilon \sup _{x}|\mathcal{H}|$, 
and

$$
\begin{aligned}
& k(t, \varepsilon) \doteq \sum_{i, j} \frac{\varepsilon^{2}}{2}\left\{\iint-\left|\gamma_{i, x}(x)\right| \operatorname{sgn}\left\langle\eta_{i}(x), \gamma_{j, x}(y)\right\rangle\left\langle\eta_{i}(x), \int_{0}^{t}\left(K_{j j}^{1}(y) V_{j}(\tau, y), K_{j j}^{2}(y) W_{j}(\tau, y)\right) d \tau\right\rangle\right. \\
&+\sum_{k \neq j}\left|\left\langle\left(-W_{i}(x), V_{i}(x)\right), \int_{0}^{t}\left(K_{j k}^{1}(y) V_{k}(\tau, y), K_{j k}^{2}(y) W_{k}(\tau, y)\right) d \tau\right\rangle\right| \\
&+\sum_{k}\left|\left\langle\left(-W_{i}(x), V_{i}(x)\right), \int_{0}^{t}\left(K_{j k}^{\sharp}(y) W_{k}(\tau, y), K_{j k}^{b}(y) V_{k}(\tau, y)\right) d \tau\right\rangle\right| \\
&-\left|\gamma_{i, x}(x)\right| \operatorname{sgn}\left\langle\eta_{i}(x), \tilde{\gamma}_{j, x}(y)\right\rangle\left\langle\eta_{i}(x), \int_{0}^{t}\left(K_{j j}^{2}(y) W_{j}(y), K_{j j}^{1}(y) V_{j}(y)\right) d \tau\right\rangle \\
&+\sum_{k \neq j}\left|\left\langle\left(-W_{i}(x), V_{i}(x)\right), \int_{0}^{t}\left(K_{j k}^{2}(y) W_{k}(\tau, y), K_{j k}^{1}(y) V_{k}(\tau, y)\right) d \tau\right\rangle\right| \\
&\left.+\sum_{k}\left|\left\langle\left(-W_{i}(x), V_{i}(x)\right), \int_{0}^{t}\left(K_{j k}^{b}(y) V_{k}(\tau, y), K_{j k}^{\sharp}(y) W_{k}(\tau, y)\right) d \tau\right\rangle\right| d x d y\right\} \\
&=\sum_{i, j}\left\{\frac{\varepsilon^{2}}{2} \iint_{0}^{t}\left\{\sum_{k=1}^{n}\left|K_{k j}^{1}\right|+\left|K_{k j}^{b}\right|\right\}\left|V_{i}(\tau, x) W_{j}(\tau, y)-W_{i}(\tau, x) V_{j}(\tau, y)\right| d \tau d y d x\right. \\
&+\frac{\varepsilon^{2}}{2} \iint_{0}^{t}\left\{\sum_{k=1}^{n}\left|K_{k j}^{1}\right|+\left|K_{k j}^{b}\right|\right\}\left|V_{i}(\tau, x) V_{j}(\tau, y)-W_{i}(\tau, x) W_{j}(\tau, y)\right| d \tau d y d x \\
& \quad+\mathcal{O}(1) \delta_{0} \varepsilon^{2} \int_{0}^{t} \sup _{x}|\mathcal{K}|(\|V(\tau)\|+\|W(\tau)\|) d \tau \\
& \quad+\mathcal{O}(1) \delta_{0} \varepsilon(\|V(t)\|+\|W(t)\|)\left(\varepsilon \int_{0}^{t}\|V(\tau)\|+\|W(\tau)\| d \tau\right) .
\end{aligned}
$$

Here, $\mu$ is a small positive constant that is $\mu=\mathcal{O}(1) \delta_{0}$.

The above estimates on (6.40), (6.41) and (6.42) are due to the properties of the matrices $\mathcal{B}, \mathcal{H}$ and $\mathcal{K}$ as stated in Lemma 6.1 and by virtue of (6.18) and Lemma 5.2. It should be noted that according to the notation in the statement of this lemma, the function $\Omega(t)$ is given by

$$
\Omega=\frac{1}{\varepsilon}(h(t, \varepsilon)+k(t, \varepsilon))+\mathcal{O}(1) \delta_{0}^{2}(\|V\|+\|W\|) .
$$

Combining (6.7), (5.18) and (6.31), we arrive at

$$
\begin{aligned}
\int_{\hat{t}}^{T} k(t, \varepsilon) d t \leq & \varepsilon(\kappa+\mu) \sum_{i, j} \int_{\hat{t}}^{T}\left(\mathcal{A}\left(\gamma_{i}(t), \gamma_{j}(t)\right)+\mathcal{A}\left(\gamma_{i}(t), \tilde{\gamma}_{j}(t)\right)\right) d \tau \\
& +\varepsilon \mathcal{O}(1) \delta_{0}\left(M_{K}+\delta_{0}\right) \int_{\hat{t}}^{T}(\|V(t)\|+\|W(t)\|) d t
\end{aligned}
$$

where $\mu=\mathcal{O}(1) \delta_{0}$ can become arbitrarily small for sufficienlty small $\delta_{0}$. By virtue of $(6.43),(6.44),(6.18)$, estimate (6.33) follows. The proof is complete. 
Turning now to estimate (6.26), let us integrate (6.32) over $[\hat{t}, T]$. Because $\beta>$ $\kappa \geq 0$ and $\mu=\mathcal{O}(1) \delta_{0}$ can become arbitrarily small, then by (3.6), we deduce

$$
\begin{aligned}
\sum_{i} \int_{\hat{t}}^{T} \int \mid W_{i, x} V_{i}- & W_{i} V_{i, x} \mid d x d t \leq \sum_{i, j}\left[\mathcal{A}\left(\gamma_{i}(\hat{t}), \gamma_{j}(\hat{t})\right)+\mathcal{A}\left(\gamma_{i}(\hat{t}), \tilde{\gamma}_{j}(\hat{t})\right)\right] \\
& +\mathcal{O}(1) \delta_{0} \sum_{j} \int_{\hat{t}}^{T}\left(\left\|\Phi_{j}(t)\right\|_{L^{1}}+\left\|\Psi_{j}(t)\right\|_{L^{1}}\right) d t+\mathcal{O}(1) \delta_{0}^{2} \\
& =\mathcal{O}(1) \delta_{0}^{2} .
\end{aligned}
$$

Thus, the change in speed, linear terms are controllable.

6.3. Length Curve functional. Here, we prove that the change in speed, quadratic terms are controllable.

$$
\int_{\hat{t}}^{T} \int_{\left|W_{i} / V_{i}\right|<3 \delta_{1}}\left|V_{i}\right|^{2}\left|\left(\frac{W_{i}}{V_{i}}\right)_{x}\right|^{2} d x d t=\mathcal{O}(1) \delta_{0}^{3}
$$

Consider the length functional $\mathcal{L}$ applied to the curve $\gamma$ to be the length of this curve. If $\gamma$ is a curve that moves along the curvature then the length is monotonically decreasing. For more details on such curves, see [1]. In order to derive (6.46), apply the length functional on the curve $\gamma_{i}$ defined on (6.27), whence

$$
\mathcal{L}_{i}(t)=\mathcal{L}\left(\gamma_{i}(t)\right)=\int \sqrt{V_{i}^{2}(t, x)+W_{i}^{2}(t, x)} d x .
$$

One can easily check that

$$
\left|\gamma_{i, x x}\right|^{2}\left|\gamma_{i, x}\right|^{2}-\left\langle\gamma_{i, x}, \gamma_{i, x x}\right\rangle^{2}=V_{i}^{4}\left|\left(\frac{W_{i}}{V_{i}}\right)_{x}\right|^{2}
$$

and

$$
\frac{\left|V_{i}\right|^{3}}{\left|\gamma_{i, x}\right|^{3}}=\frac{1}{\left(1+\left(\frac{W_{i}}{V_{i}}\right)^{2}\right)^{3 / 2}}
$$


Integrating by parts, employing the above identities and the estimates in Lemma 5.2 , one can get

$$
\begin{aligned}
& \frac{d}{d t} \mathcal{L}_{i}(t)=\int \frac{\left\langle\gamma_{i, x}, \gamma_{i, x t}\right\rangle}{\sqrt{\left\langle\gamma_{i, x}, \gamma_{i, x}\right\rangle}} d x \\
& =\int \frac{\left\langle\gamma_{i, x}, \gamma_{i, x x}\right\rangle}{\left|\gamma_{i, x}\right|}-\frac{\left\langle\gamma_{i, x},\left(\tilde{\lambda}_{i} \gamma_{i, x}\right)_{x}\right\rangle}{\left|\gamma_{i, x}\right|}+\frac{\left\langle\gamma_{i, x},\left(\phi_{i}, \psi_{i}\right)\right\rangle}{\left|\gamma_{i, x}\right|} d x \\
& -\int \frac{\left\langle\gamma_{i, x}, \varepsilon\left(\sum_{k}\left(B_{i k}^{1} V_{k}+B_{i k}^{\sharp} W_{k}\right), \sum_{k}\left(B_{i k}^{b} V_{k}+B_{i k}^{2} W_{k}\right)\right)\right\rangle}{\left|\gamma_{i, x}\right|} d x \\
& +\int \frac{\left\langle\gamma_{i, x}, \varepsilon\left(\sum_{k}\left(H_{i k}^{1} V_{0, k}+H_{i k}^{\sharp} W_{0, k}\right), \sum_{k}\left(H_{i k}^{b} V_{0, k}+H_{i k}^{2} W_{0, k}\right)\right)\right\rangle}{\left|\gamma_{i, x}\right|} d x \\
& -\int \frac{\left\langle\gamma_{i, x}, \varepsilon^{2}\left(\sum_{k} \int_{0}^{t}\left(K_{i k}^{1} V_{k}(\tau)+K_{i k}^{\sharp} W_{k}(\tau)\right) d \tau, \sum_{k} \int_{0}^{t}\left(K_{i k}^{b} V_{k}(\tau)+K_{i k}^{2} W_{k}(\tau)\right) d \tau\right)\right\rangle}{\left|\gamma_{i, x}\right|} d x \\
& \leq-\int \frac{\left|V_{i}\right|\left|\left(\frac{W_{i}}{V_{i}}\right)_{x}\right|^{2}}{\left(1+\left(\frac{W_{i}}{V_{i}}\right)^{2}\right)^{3 / 2}} d x+\left\|\Phi_{i}(t)\right\|_{L^{1}}+\left\|\Psi_{i}(t)\right\|_{L^{1}}+\varepsilon \delta_{0} \sup _{x}|\mathcal{H}| \\
& +\varepsilon \int\left(\sum_{k}\left|B_{i k}^{1}\right|\left|V_{k}\right|+\sum_{k}\left|B_{i k}^{\sharp}\right|\left|W_{k}\right|+\sum_{k}\left|B_{i k}^{b}\right|\left|V_{k}\right|+\sum_{k}\left|B_{i k}^{2}\right|\left|W_{k}\right|\right) d x \\
& +\varepsilon^{2} \int_{0}^{t} \int \sum_{k}\left(\left|K_{i k}^{1}\right|\left|V_{k}(\tau)\right|+\left|K_{i k}^{\sharp}\right|\left|W_{k}(\tau)\right|+\left|K_{i k}^{\mathrm{b}}\right|\left|V_{k}(\tau)\right|+\left|K_{i k}^{2}\right|\left|W_{k}(\tau)\right|\right) d x d \tau \\
& \leq-\frac{1}{\left(1+9 \delta_{1}^{2}\right)^{3 / 2}} \int_{\left|\frac{W_{i}}{V_{i}}\right| \leq 3 \delta_{1}}\left|V_{i}\right|\left|\left(\frac{W_{i}}{V_{i}}\right)_{x}\right|^{2} d x+\left\|\Phi_{i}(t)\right\|_{L^{1}}+\left\|\Psi_{i}(t)\right\|_{L^{1}} \\
& +\varepsilon M_{B} \sum_{k}\left(\left\|V_{k}\right\|_{L^{1}}+\left\|W_{k}\right\|_{L^{1}}\right)+\varepsilon \delta_{0} \sup _{x}|\mathcal{H}| \\
& +\varepsilon^{2} \int_{0}^{t} \int\left(\sum_{k}\left(\left|K_{i k}^{1}\right|\left|V_{k}(\tau)\right|+\left|K_{i k}^{\sharp}\right|\left|W_{k}(\tau)\right|+\left|K_{i k}^{b}\right|\left|V_{k}(\tau)\right|+\left|K_{i k}^{2}\right|\left|W_{k}(\tau)\right|\right) d x d \tau .\right.
\end{aligned}
$$

Therefore, (5.19) implies

$$
\begin{aligned}
& \int_{\left|\frac{W_{i}}{V_{i}}\right| \leq 3 \delta_{1}}\left|V_{i}\right|^{2}\left|\left(\frac{W_{i}}{V_{i}}\right)_{x}\right|^{2} d x \leq \delta_{0}^{2} \int_{\left|\frac{W_{i}}{V_{i}}\right| \leq 3 \delta_{1}}\left|V_{i}\right|\left|\left(\frac{W_{i}}{V_{i}}\right)_{x}\right|^{2} d x \\
& \leq \delta_{0}^{2}\left(1+9 \delta_{1}^{2}\right)^{3 / 2}\left[-\frac{d}{d t} \mathcal{L}_{i}(t)+\mathcal{O}(1) \varepsilon \sum_{k}\left(\left\|V_{k}\right\|+\left\|W_{k}\right\|\right)+\mathcal{O}(1) \delta_{0} \varepsilon \sup _{x}|\mathcal{H}|\right. \\
& \left.+\varepsilon^{2} \int_{0}^{t} \int \sum_{k}\left(\left|K_{i k}^{1}\right|\left|V_{k}(\tau)\right|+\left|K_{i k}^{\sharp}\right|\left|W_{k}(\tau)\right|+\left|K_{i k}^{b}\right|\left|V_{k}(\tau)\right|+\left|K_{i k}^{2}\right|\left|W_{k}(\tau)\right|\right) d x d \tau\right] .
\end{aligned}
$$

Consequently, integrating the above inequality over $[\hat{t}, T],(6.46)$ follows. Indeed,

$$
\varepsilon \int_{\hat{t}}^{T}\left\|V_{k}(t)\right\|+\left\|W_{k}(t)\right\| d t=\mathcal{O}(1) \delta_{0}
$$


and the last term can be written in the form

$$
\begin{aligned}
& \varepsilon^{2} \int_{\hat{t}}^{T}\left(\int_{\tau}^{T} \sup _{x}\left|K_{i k}^{1}\right|+\sup _{x}\left|K_{i k}^{b}\right| d t\right)\left\|V_{k}(\tau)\right\|_{L^{1}} d \tau \\
& \quad+\varepsilon^{2} \int_{0}^{t}\left(\int_{\hat{t}}^{T}\left(\sup _{x}\left|K_{i k}^{1}\right|+\sup _{x}\left|K_{i k}^{b}\right| d t\right)\left\|V_{k}(\tau)\right\|_{L^{1}} d \tau=\mathcal{O}(1) M_{K} \delta_{0} .\right.
\end{aligned}
$$

Similarly the $W_{k}$ expression. By virtue of (5.18) and changing the order of integration, the result follows. Thus we actually get a cubic power of the total variation of initial data for this type of terms.

6.4. Energy estimates. In this subsection, we show that the cutoff terms in (6.9) are controllable, i.e.

$$
\int_{\hat{t}}^{T} \int\left(\left|V_{i, x}\right|+\left|W_{i, x}\right|\right)\left|W_{i}-\theta_{i} V_{i}\right| d x d t=\mathcal{O}(1) \delta_{0}^{2} .
$$

We proceed in the same way as in $[3,7]$. In other words, the aim is to reduce the integrand of (6.48) into controllable terms and other new terms and to treat the latter by means of energy methods. To achieve this, we first establish some useful relations between $V_{i}, W_{i}$ and $V_{i, x}$. Consider the cutoff functions $\eta, \zeta: \mathbb{R} \rightarrow[0,1]$

$$
\eta(s)=\left\{\begin{array}{ll}
0 & |s| \leq \frac{3 \delta_{1}}{5} \\
1 & |s| \geq \frac{4 \delta_{1}}{5}
\end{array} \quad \zeta(s)=\eta\left(|s|-\frac{\delta_{1}}{5}\right) \leq \eta(s)\right.
$$

For shorthand, we will be using $\eta_{i}=\eta\left(\frac{w_{i}}{v_{i}}\right)$ and $\zeta_{i}=\zeta\left(\frac{w_{i}}{v_{i}}\right)$. The following lemma is the tool needed to express the cutoff terms in (6.48) in terms of quantities that are easier to handle.

Lemma 6.5. If $\left|W_{i} / V_{i}\right| \geq 3 \delta_{1} / 5$, then

$$
\begin{array}{r}
\left|W_{i}\right| \leq 2\left|V_{i, x}\right|+\mathcal{O}(1) \cdot \delta_{0} \sum_{j \neq i}\left(\left|V_{j}\right|+\left|V_{j, x}\right|+\left|W_{j}\right|\right), \\
\left|V_{i}\right| \leq \frac{5}{2 \delta_{1}}\left|V_{i, x}\right|+\mathcal{O}(1) \cdot \delta_{0} \sum_{j \neq i}\left(\left|V_{j}\right|+\left|V_{j, x}\right|+\left|W_{j}\right|\right) .
\end{array}
$$

If $\left|w_{i} / v_{i}\right| \leq \delta_{1}$, then

$$
\left|V_{i, x}\right| \leq 2 \delta_{1}\left|V_{i}\right|+\mathcal{O}(1) \cdot \delta_{0} \sum_{j \neq i}\left(\left|V_{j}\right|+\left|V_{j, x}\right|+\left|W_{j}\right|\right) .
$$

Proof. Combining equation (2.11) and the decomposition (5.10), yields

$$
\sum_{i}\left(W_{i}-\lambda_{i}^{*} V_{i}\right) \tilde{r}_{i}+\sum_{i} V_{i} A(U) \tilde{r}_{i}=\sum_{i} V_{i, x} \tilde{r}_{i}+\sum_{i} V_{i}\left[\sum_{j} V_{j} \tilde{r}_{i, U} \tilde{r}_{j}+V_{i, x} \tilde{r}_{i, V}-\theta_{i, x} \tilde{r}_{i, \sigma}\right] .
$$

Taking the inner product with $\tilde{r}_{i}$, one obtains

$$
V_{i, x}=W_{i}+\left(\tilde{\lambda}_{i}-\lambda_{i}^{*}\right) V_{i}+\Theta_{i}
$$


where

$$
\begin{aligned}
\Theta_{i}= & \left.\sum_{j \neq i}\left(W_{j}-\lambda_{j}^{*} V_{j}\right)\left\langle\tilde{r}_{i}, \tilde{r}_{j}\right\rangle+\sum_{j \neq i}\left\langle\tilde{r}_{i}, A(U)\right) \tilde{r}_{j}\right\rangle V_{j}-\sum_{j \neq i} \sum_{k}\left\langle\tilde{r}_{i}, \tilde{r}_{j, U} \tilde{r}_{k}\right\rangle V_{j} V_{k} \\
& -\sum_{j \neq i}\left\langle\tilde{r}_{i}, \tilde{r}_{j, V}\right\rangle V_{j} V_{j, x}+\sum_{j \neq i}\left\langle\tilde{r}_{i}, \tilde{r}_{j, \sigma}\right\rangle V_{j} \theta_{j, x}-\sum_{j \neq i}\left\langle\tilde{r}_{i}, \tilde{r}_{j}\right\rangle V_{j, x} \\
(6.54)= & \mathcal{O}(1) \cdot \delta_{0} \sum_{j \neq i}\left(\left|V_{j}\right|+\left|V_{j, x}\right|+\left|W_{j}\right|\right) .
\end{aligned}
$$

From this point and on, one can establish the bounds by carefully studying (6.53). The proof can be completed by applying the same arguments as in Lemma 6.6 in [7], since the relation (6.53) is the same as in [7] because of the choice of (5.10).

Now, observe that the quantity $\left|W_{i}-\theta_{i} V_{i}\right|$ vanishes when $\left|W_{i} / V_{i}\right| \leq \delta_{1}$. Otherwise, $\left|W_{i}-\theta_{i} V_{i}\right| \leq\left|W_{i}\right|$. By means of the cutoff function $\zeta$ and the relation (6.50), one has

$$
\left|W_{i}-\theta_{i} V_{i}\right| \leq\left|\zeta_{i} W_{i}\right| \leq \zeta_{i}\left(2\left|V_{i, x}\right|+\mathcal{O}(1) \delta_{0} \sum_{j \neq i}\left(\left|V_{j}\right|+\left|V_{j, x}\right|+\left|W_{j}\right|\right)\right),
$$

which implies that

$$
\begin{gathered}
\left(\left|V_{i, x}\right|+\left|W_{i, x}\right|\right)\left|W_{i}-\theta_{i} V_{i}\right| \leq\left(\left|V_{i, x}\right|+\left|W_{i, x}\right|\right) \zeta_{i}\left(2\left|V_{i, x}\right|+\mathcal{O}(1) \delta_{0} \sum_{j \neq i}\left|V_{j}\right|+\left|V_{j, x}\right|+\left|W_{j}\right|\right) \\
\leq 3 \eta_{i} V_{i, x}^{2}+\zeta_{i} W_{i, x}^{2}+\mathcal{O}(1) \cdot \delta_{0} \sum_{j \neq i}\left(\left|V_{i, x}\right|+\left|W_{i, x}\right|\right)\left(\left|V_{j}\right|+\left|V_{j, x}\right|+\left|W_{j}\right|\right)
\end{gathered}
$$

by Schwarz's inequality. Because all transveral terms are controllable, in order to prove (6.48), it suffices to prove

$$
\int_{\hat{t}}^{T} \int \eta_{i} V_{i, x}^{2} d x d t=\mathcal{O}(1) \delta_{0}^{2}, \quad \int_{\hat{t}}^{T} \int \zeta_{i} W_{i, x}^{2} d x d t=\mathcal{O}(1) \cdot \delta_{0}^{2}
$$

for all $i=1, \ldots, n$.

Here, we choose to present only the energy estimate (6.56.1), since the corresponding estimate $(6.56 .2)$ follows similarly. To begin with, we multiply the evolution equation of $V_{i}$ given in $(6.1)$ by $\eta_{i} V_{i}$ and integrate by parts, to get

$$
\begin{aligned}
\int \eta_{i} V_{i, x}^{2} d x= & -\int\left(\eta_{i} \frac{V_{i}^{2}}{2}\right)_{t}+\varepsilon \sum_{k} \eta_{i} V_{i}\left(B_{i k}^{1} V_{k}+B_{i k}^{\sharp} W_{k}\right) d x \\
& +\varepsilon \int \sum_{k} \eta_{i} V_{i}\left(H_{i k}^{1} V_{0, k}+H_{i k}^{\sharp} W_{0, k}\right) d x \\
& -\varepsilon^{2} \int \sum_{k} \eta_{i} V_{i} \int_{0}^{t}\left(K_{i k}^{1} V_{k}(\tau)+K_{i k}^{\sharp} W_{k}(\tau)\right) d \tau d x \\
& +\int\left(\eta_{i, t}+\tilde{\lambda}_{i} \eta_{i, x}-\eta_{i, x x}\right) \frac{V_{i}^{2}}{2} d x \\
& -\int \eta_{i} \tilde{\lambda}_{i, x} \frac{V_{i}^{2}}{2} d x-2 \int \eta_{i, x} V_{i} V_{i, x} d x+\int \eta_{i} V_{i} \Phi_{i} d x
\end{aligned}
$$


Upon employing the evolution equation of $\eta_{i}$, we can treat all non- $\varepsilon$ terms of (6.57) in the same way as in [7]. However, it worths explaining the treatment of the $\varepsilon$-terms in (6.57), that distinguish these estimates here from those in [7]. It turns out that the three terms that have a factor of $\varepsilon$ or $\varepsilon^{2}$ in (6.57) are bounded by

$$
\mathcal{O}(1) \delta_{0}^{2} M_{B} \varepsilon(\|V\|+\|W\|), \quad \mathcal{O}(1) \delta_{0}^{3} \varepsilon \sup _{x}|\mathcal{H}|, \quad \mathcal{O}(1) \delta_{0}^{2} M_{K} \varepsilon\|V\|,
$$

respectively. By virtue of (5.18) and (6.18), all above terms are integrable over $[\hat{t}, T]$ and thus, it implies the validity of (6.56.1).

To sum up, the four estimates (6.22), (6.26), (6.46) and (6.48) together with Lemma 6.1 prove Lemma 3.1. Thus, we showed that the solution $U$ is globally defined having bounded BV norm, which is also in $L^{1}[0,+\infty)$, i.e.

$$
T V\{U(t)\}+\varepsilon \int_{0}^{t} T V\{U(\tau)\} d \tau=\mathcal{O}(1) T V\{\bar{U}\}
$$

for all $t>0$. It worths noting that there is actually a factor $(\beta-\kappa)$ in front of the integral in (6.58), which we omit. More precisely, $\mathcal{O}(1)$ depends on $1 /(\beta-\kappa)$ because of the integrability of $\varepsilon\|V(t)\|$ and $\varepsilon\|W(t)\|$ over $[0, \infty)$.

\section{Stability}

In this section, we establish the stability of solutions to (7.1)

$$
\begin{aligned}
U_{t}+A(U) U_{x}-U_{x x}+\varepsilon g(U)= & \varepsilon H(\varepsilon t) U_{0}-\varepsilon^{2} \int_{0}^{t} K(\varepsilon(t-\tau)) U(\tau) d \tau+U_{x x}, \\
& U(0, x)=U_{0}(x) .
\end{aligned}
$$

Let $Z(t, x)$ be the infinitesimal perturbation of $U$, then $Z$ satisfies the following equation:

$$
\begin{gathered}
Z_{t}+(A(U) Z)_{x}+\varepsilon D g(U) Z-Z_{x x}=\varepsilon H(\varepsilon t) Z_{0}-\varepsilon^{2} \int_{0}^{t} K(\varepsilon(t-\tau)) Z(\tau) d \tau \\
+\left(U_{x} \bullet A(U)\right) Z-(Z \bullet A(U)) U_{x} \\
Z(0, x)=Z_{0}(x) .
\end{gathered}
$$

Lemma 7.1. If $Z$ is the infinitesimal perturbation of $U$ then it satisfies the following bounds

$$
\|Z(t)\|_{L^{1}}+\varepsilon \int_{0}^{t}\|Z(\tau)\|_{L^{1}} d \tau \leq L\left\|Z_{0}\right\|_{L^{1}}
$$

for some constant $L$ independent of $\varepsilon$.

We claim that the above lemma implies the stability of solutions $U$ to (7.1), by employing the standard homotopy argument of Bressan. Indeed, we consider the path of initial data

$$
U_{0}^{\theta}(x)=\theta U_{0}(x)+(1-\theta) V_{0}(x)
$$

and let $U^{\theta}$ denote the solution to (7.1) with initial data $U_{0}^{\theta}$. By direct calulations, one can verify that the tangent vector $Z^{\theta}(t, x)=\frac{d U^{\theta}}{d \theta}$ to the path $\theta \mapsto U^{\theta}$, for $\theta \in[0,1]$, is the corresponding infinitesimal perturbation to $U^{\theta}$ with initial data

$$
Z^{\theta}(0, x)=U_{0}(x)-V_{0}(x) .
$$


Therefore, by Lemma 7.1, it follows

$$
\begin{aligned}
\|U(t)-V(t)\|_{L^{1}}+\varepsilon & \int_{0}^{t}\|U(\tau)-V(\tau)\|_{L^{1}} d \tau \leq \\
& \leq \int_{0}^{1}\left[\left\|\frac{d U^{\theta}}{d \theta}(t)\right\|_{L^{1}}+\varepsilon \int_{0}^{t}\left\|\frac{d U^{\theta}}{d \theta}(\tau)\right\|_{L^{1}}\right] d \theta \\
& \leq L \int_{0}^{1}\left\|U_{0}-V_{0}\right\|_{L^{1}} d \theta=L\left\|U_{0}-V_{0}\right\|_{L^{1}}
\end{aligned}
$$

Thus, the claim holds. This establishes that the solutions to parabolic system (7.1) are uniformly stable in $L^{1}$ and moreover that the $L^{1}$ distance of two solutions tends to zero as $t \rightarrow+\infty$. The argument is similar to the one used in $[3,7]$, however here because of the presence of the memory term that induces dissipation, the integrability with respect to time can further be established. Note that in [7], $K=H=0$, hence a stronger result holds, i.e. $\|Z(t)\|_{L^{1}}$ decays exponentially in time.

In view of the above discussion, it remains to prove Lemma 7.1. Recall that in Section 4, (7.3) is established for $t \in[0, \hat{t}]$. The proof of $(7.3)$ for greater times retraces the steps followed in Sections 5-6 to get the BV bounds. Note that if $Z=U_{x}$ then (7.3) reduces to (2.12). Therefore, in the author's opinion it is enough to outline how this procedure can be extended to establish (7.3) and omit the details.

First, we define the flux $Y$ of $Z$ by

$$
Y=Z_{x}-A(U) Z \text {. }
$$

The aim is to decompose $(Z, Y)$ pointwise along a suitable basis so that the decomposition must be compatible with (5.10), when $Z=U_{x}$. Given a solution $U$ and the corresponding components $V_{i}$ of the decomposition (5.10), we seek $(P, Q) \in \mathbb{R}^{2 n}$ so that

$$
\begin{aligned}
& Z=\sum_{i=1}^{n} P_{i} \tilde{r}_{i}\left(U, V_{i}, \lambda_{i}^{*}-\theta\left(\frac{Q_{i}}{P_{i}}\right)\right), \\
& Y=\sum_{i=1}^{n}\left(Q_{i}-\lambda_{i}^{*} P_{i}\right) \tilde{r}_{i}\left(U, V_{i}, \lambda_{i}^{*}-\theta\left(\frac{Q_{i}}{P_{i}}\right)\right),
\end{aligned}
$$

where $\tilde{r}_{i}$ are the vectors defined in Section 5 . We use the standard notation introduced [3]:

$$
\begin{gathered}
\hat{r}_{i} \doteq \tilde{r}_{i}\left(U, V_{i}, \lambda_{i}^{*}-\theta\left(\frac{Q_{i}}{P_{i}}\right)\right), \\
\hat{\theta}_{i} \doteq \theta\left(\frac{Q_{i}}{P_{i}}\right) \quad \text { and } \quad \hat{\lambda}_{i} \doteq\left\langle\hat{r}_{i}, A(u) \hat{r}_{i}\right\rangle .
\end{gathered}
$$

We can show that there exist unique $P$ and $Q$ so that the decomposition (7.8) holds and get the corresponding result to Lemma 5.1. Also, we get $L^{1}$ estimates on the perturbed components $P_{i}$ and $Q_{i}$ in the same way already treated for $V_{i}$ and $W_{i}$. (See also Lemma 7.2 and 7.3 in [7]). Having achieved that, the next step is to study the evolution of the components. As before, we show that they satisfy a $2 n \times 2 n$ hyperbolic system with source. The following lemma states these results. 
Lemma 7.2. The decomposition (7.8) holds for $\left|U-U^{*}\right|$ and $|V|$ small. If the bound (7.3) holds for $t \in[0, T]$, then for all $t \in[\hat{t}, T]$ the perturbed components $P_{i}$, $Q_{i}$ satisfy the following estimates

$$
\begin{aligned}
\left\|P_{i}(t)\right\|_{L^{1}},\left\|Q_{i}(t)\right\|_{L^{1}}, \varepsilon \int_{0}^{t}\left\|P_{i}(\tau)\right\|_{L^{1}} d \tau, \varepsilon \int_{0}^{t}\left\|Q_{i}(\tau)\right\|_{L^{1}} d \tau & =\mathcal{O}(1) \delta_{0} \\
\left\|P_{i}(t)\right\|_{L^{\infty}},\left\|Q_{i}(t)\right\|_{L^{\infty}},\left\|P_{i, x}(t)\right\|_{L^{1}},\left\|Q_{i, x}(t)\right\|_{L^{1}} & =\mathcal{O}(1) \delta_{0}^{2} \\
\left\|P_{i, x}(t)\right\|_{L^{\infty}}, \quad\left\|Q_{i, x}(t)\right\|_{L^{\infty}} & =\mathcal{O}(1) \delta_{0}^{3}
\end{aligned}
$$

The unique solution $(P, Q)$ of the decomposition (7.8) satisfies a $2 n \times 2 n$ viscous hyperbolic system of balance laws with source of the form

$$
\begin{array}{r}
\left(\begin{array}{l}
P \\
Q
\end{array}\right)_{t}+\left[\left(\begin{array}{cc}
\Lambda & 0 \\
0 & \Lambda
\end{array}\right)\left(\begin{array}{l}
P \\
Q
\end{array}\right)\right]_{x}-\left(\begin{array}{l}
P \\
Q
\end{array}\right)_{x x}+\varepsilon\left(\begin{array}{ll}
\widehat{B^{1}} & \widehat{B^{\sharp}} \\
\widehat{B^{b}} & \widehat{B^{2}}
\end{array}\right)\left(\begin{array}{l}
P \\
Q
\end{array}\right)=\varepsilon\left(\begin{array}{ll}
\widehat{H^{1}} & \widehat{H^{\sharp}} \\
\widehat{H^{b}} & \widehat{H^{2}}
\end{array}\right)\left(\begin{array}{c}
P_{0} \\
Q_{0}
\end{array}\right) \\
\quad-\varepsilon^{2} \int_{0}^{t}\left(\begin{array}{cc}
\widehat{K^{1}} & \widehat{K^{\sharp}} \\
\widehat{K^{b}} & \widehat{K^{2}}
\end{array}\right)\left(\begin{array}{l}
P(\tau) \\
Q(\tau)
\end{array}\right) d \tau+\left(\begin{array}{c}
\widehat{\Phi} \\
\widehat{\Psi}
\end{array}\right),
\end{array}
$$

where $\Lambda$ is the $n \times n$ diagonal matrix with entries $\left\{\tilde{\lambda}_{i}\right\}$, the matrix $\left(\frac{\widehat{B^{1}}}{\widehat{B^{b}}} \widehat{B^{\sharp}}\right)$ is diagonally dominant, the matrix $\left(\widehat{\widehat{H^{1}}} \widehat{\widehat{H^{\natural}}} \widehat{H^{2}}\right)$ is in $L^{1}\left(M_{2 n \times 2 n} ;[0,+\infty)\right)$ and the matrix

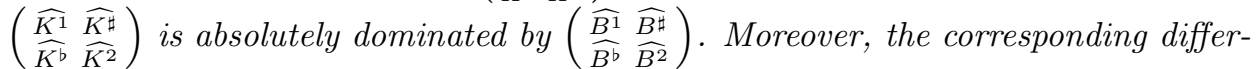
ences to those in (6.2)-(6.4) are of order $\mathcal{O}\left(\left|U-U^{*}\right|,|V|\right)$ and the corresponding estimates to those (6.5)-(6.8) hold, e.g.

$$
\varepsilon \int_{0}^{+\infty} \sum_{j}\left|\widehat{K_{j i}^{1}}(\varepsilon s)\right|+\left|\widehat{K_{j i}^{b}}(\varepsilon s)\right| d s<\kappa+\mathcal{O}(1) \delta_{0} .
$$

Furthermore, the source terms in (7.14) satisfy the bounds

$$
\begin{aligned}
& \widehat{\Phi}_{i}, \widehat{\Psi}_{i}=\mathcal{O}(1) \cdot \sum_{j \neq i}\left(\left|P_{i} V_{j}\right|+\left|Q_{i} V_{j}\right|+\left|P_{i} V_{j, x}\right|+\left|Q_{i} V_{j, x}\right|+\left|P_{i} W_{j}\right|+\left|Q_{i} W_{j}\right|\right. \\
& \quad+\left|P_{i, x} W_{j}\right|+\left|Q_{i, x} W_{j}\right|+\left|P_{i, x} V_{j}\right|+\left|Q_{i, x} V_{j}\right|+\left|P_{i, x} V_{j, x}\right|+\left|Q_{i, x} V_{j, x}\right|+\left|P_{i} Q_{j, x}\right| \\
& \left.\quad+\left|P_{i} P_{j, x}\right|+\left|P_{i, x} Q_{j, x}\right|+\left|P_{i, x} P_{j, x}+\right| Q_{i} P_{j, x}|+| Q_{i} Q_{j, x} \mid\right) \longrightarrow \text { Transversal } \\
& +\mathcal{O}(1) \cdot \sum_{j}\left(\left|P_{j, x} W_{j}-P_{j} W_{j, x}\right|+\left|Q_{j, x} W_{j}-Q_{j} W_{j, x}\right|+\left|Q_{i, x} P_{i}-P_{i, x} Q_{i}\right|\right. \\
& \left.\quad+\left|P_{j, x} V_{j}-P_{j} V_{j, x}\right|+\left|Q_{j, x} V_{j}-Q_{j} V_{j, x}\right|\right) \longrightarrow \text { Change in speed, Linear } \\
& +\mathcal{O}(1) \cdot \sum_{j}\left(\left|V_{j}\right|+\left|P_{j}\right|\right)\left|P_{j}\right|\left|\left(\frac{Q_{j}}{P_{j}}\right)_{x}\right|^{2} \chi_{\left\{\left|\frac{Q_{j}}{P_{j}}\right|<3 \delta_{1}\right\}} \rightarrow \text { Change in speed, Quadr. } \\
& +\mathcal{O}(1) \cdot \sum_{j}\left(\left|P_{j, x}\right|+\left|Q_{j, x}\right|+\left|P_{j} V_{j}\right|+\left|Q_{j} V_{j}\right|\right) \cdot\left|W_{j}-\theta_{j} V_{j}\right| \longrightarrow \text { Cutoff } \\
& +\mathcal{O}(1) \cdot \sum_{j}\left|P_{j} \Phi_{j}\right|+\left|Q_{j} \Phi_{j}\right| \longrightarrow \text { Source of gradient component } V
\end{aligned}
$$

$$
+\mathcal{O}(1) \cdot \varepsilon \sum_{j, k}\left(\left|P_{j} V_{k}\right|+\left|P_{j} W_{k}\right|+\left|Q_{j} V_{k}\right|+\left|Q_{j} W_{k}\right|\right) . \rightarrow \varepsilon-\text { Order Term }
$$


Proof of Lemma 7.1. The estimate follows easily by combining the next lemma and the standard continuation argument as employed in Section 3 below Lemma 3.1 to establish BV bounds.

Lemma 7.3. Let $Z$ be a solution of (7.2) such that for $t \in[\hat{t}, T], Z$ satisfies

$$
\|Z(t)\|+\varepsilon \int_{0}^{t}\|Z(\tau)\| d \tau \leq \delta_{0}
$$

Suppose the perturbed source terms $\widehat{\Phi}$ and $\widehat{\Psi}$ that appear in (7.14) satisfy

$$
\int_{\hat{t}}^{T} \int \sum_{i=1}^{n}\left(\left|\widehat{\Phi}_{i}(t, x)\right|+\left|\widehat{\Psi}_{i}(t, x)\right|\right) d x d t \leq \delta_{0}
$$

then the following estimate holds

$$
\int_{\hat{t}}^{T} \int \sum_{i=1}^{n}\left(\left|\widehat{\Phi}_{i}(t, x)\right|+\left|\widehat{\Psi}_{i}(t, x)\right|\right) d x d t=\mathcal{O}(1) \delta_{0}^{2},
$$

for sufficiently small $\delta_{0}$.

Proof. By employing the interaction potential function $\mathcal{Q}$, the area functional $\mathcal{A}$, the length curve functional $\mathcal{L}$ and the energy functionals as well as the techniques implemented in Sections 6.1-6.4, one can treat all perturbed source terms that belong to the first four categories in (7.16). However, one should utilize the more general form of the planar curves $\gamma$ and $\tilde{\gamma}$ as described in [7]:

$$
\gamma_{i}^{(v, p)}(t, x)=\left(\int_{-\infty}^{x} v_{i}(t, y) d y, \int_{-\infty}^{x} p_{i}(t, y) d y\right)
$$

and similarly $\gamma_{i}^{(v, q)}(t, x), \gamma_{i}^{(w, p)}(t, x), \gamma_{i}^{(w, q)}(t, x), \gamma_{i}^{(p, q)}(t, x)$ etc. Therefore, one should apply the area and length functionals on these generalized curves, e.g. $\mathcal{A}\left(\gamma_{i}^{(v, p)}, \gamma_{j}^{(v, p)}\right), \mathcal{L}\left(\gamma_{i}^{(v, p)}(t)\right)$.

The terms in the last two categories in (7.16) are controllable. Indeed, by Lemma 3.1 and $(7.12)$, it follows

$$
\int_{\hat{t}}^{T} \int\left(\left|P_{i} \Phi_{i}\right|+\left|Q_{i} \Phi_{i}\right|\right) d x d t=\mathcal{O}(1) \cdot \delta_{0}^{3},
$$

for all $i=1, \ldots, n$, and combining Lemmas 5.2 and 7.2 , we also arrive at

$$
\varepsilon \int_{\hat{t}}^{T} \int\left(\left|P_{j} V_{k}\right|+\left|P_{j} W_{k}\right|+\left|Q_{j} V_{k}\right|\right) d x d t=\mathcal{O}(1) \cdot \delta_{0}^{3},
$$

for all $j, k=1, \ldots, n$. Thus, all terms in (7.16) are controllable, i.e. satisfy (7.19).

\section{Convergence of approximate solutions.}

Up to this point, we proved that solution $U(t, x)$ to

$$
\begin{aligned}
& U_{t}+A(U) U_{x}+\varepsilon g(U)=\varepsilon H(\varepsilon t) U_{0}-\varepsilon^{2} \int_{0}^{t} K(\varepsilon(t-\tau)) U(\tau) d \tau+U_{x x} \\
& U(0, x)=U_{0}(x)=\bar{U}(\varepsilon x),
\end{aligned}
$$


is globally defined in time, the total variarion is uniformly bounded according to

$$
T V\{U(t)\}+\varepsilon \int_{0}^{\infty} T V\{U(s)\} d s \leq C T V \bar{U}, \quad \text { for all } t
$$

and the solution is uniformly stable with respect to initial data:

$$
\|U(t)-V(t)\|_{L^{1}}+\varepsilon \int_{0}^{\infty}\|U(\tau)-V(\tau)\| d \tau \leq L\left\|U_{0}-V_{0}\right\|_{L^{1}}
$$

An immediate implementation of the stability estimate is a uniform $L^{1}$ bound

$$
\left\|U(t)-U^{*}\right\|_{L^{1}}+\varepsilon \int_{0}^{t}\left\|U(\tau)-U^{*}\right\| d \tau \leq L\left\|U_{0}-U^{*}\right\|_{L^{1}}=L \frac{1}{\varepsilon}\left\|\bar{U}-U^{*}\right\|_{L^{1}},
$$

where $U^{*}$ is the equilibrium.

Now, to investigate the continuous dependence of $U$ in time, it suffices to combine the above estimates. Indeed, by Proposition 4.2 and (8.3), we get

$$
\left\|U_{x}(t)\right\|_{L^{1}} \leq C \delta_{0} \quad \text { for all } t>0, \quad\left\|U_{x x}(t)\right\|_{L^{1}} \leq \begin{cases}\frac{2 k \delta_{0}}{\sqrt{t}} & \text { for } t<\hat{t} \\ \frac{2 k \delta_{0}}{\sqrt{\hat{t}}} & \text { for } t \geq \hat{t}\end{cases}
$$

Combining equation (8.1) and estimate (8.5), we arrive at imply

$$
\begin{aligned}
\left\|U_{t}(t)\right\|_{L^{1}} & \leq\left\|U_{x x}(t)\right\|_{L^{1}}+\left\|A(U) U_{x}\right\|_{L^{1}} \\
& +\varepsilon \mathcal{O}(1)\left(\left\|U(t)-U^{*}\right\|_{L^{1}}+\left\|U_{0}-U^{*}\right\|_{L^{1}}+\varepsilon \int_{0}^{t}\left\|U(\tau)-U^{*}\right\|_{L^{1}} d \tau\right) \\
& \leq L^{\prime}\left(1+\frac{1}{2 \sqrt{t}}\right),
\end{aligned}
$$

for an appropriate constant $L^{\prime}$. For fixed times $t>s \geq 0$, by integrating the above estimate over $[s, t]$, we deduce

$$
\begin{aligned}
\|U(t)-U(s)\|_{L^{1}} & \leq \int_{s}^{t}\left\|U_{t}(\tau)\right\|_{L^{1}} d \tau \\
& \leq L^{\prime}(|t-s|+|\sqrt{t}-\sqrt{s}|) .
\end{aligned}
$$

Our results so far refer to the solution of the parabolic system (8.1)-(8.2). However, in view of the discussion in Section 2, by rescaling the coordinates, our analysis can be extended to the vanishing viscosity approximations $U^{\varepsilon}=U^{\varepsilon}(t, x)$. Consider the viscous hyperbolic system (2.5)-(2.6) and recall that

$$
U^{\varepsilon}(t, x)=U\left(\frac{t}{\varepsilon}, \frac{x}{\varepsilon}\right),
$$

where $U$ satisfies (8.1)-(8.2). Consequently, parabolic system (2.5)-(2.6) has a unique solution $U^{\varepsilon}$ defined for all $t>0$ and

$$
\begin{aligned}
T V\left\{U^{\varepsilon}(t)\right\}+\int_{0}^{t} T V\left\{U^{\varepsilon}(\tau)\right\} d \tau & =T V\{U(t / \varepsilon)\}+\varepsilon \int_{0}^{t / \varepsilon} T V\{U(\tau)\} d \tau \\
& \leq T V\{U(t / \varepsilon)\}+\varepsilon \int_{0}^{\infty} T V\{U(\tau)\} d \tau \\
& \leq C T V\{\bar{U}\} .
\end{aligned}
$$


If $V^{\varepsilon}$ is another solution to (2.5) with initial data $\bar{V} \in L^{1}$, then by (8.4), we obtain the stability of solutions to $(2.5)$ :

$$
\begin{aligned}
& \left\|U^{\varepsilon}(t)-V^{\varepsilon}(t)\right\|_{L^{1}}+\int_{0}^{t}\left\|U^{\varepsilon}(s)-V^{\varepsilon}(s)\right\|_{L^{1}} d s= \\
& =\varepsilon\|U(t / \varepsilon)-V(t / \varepsilon)\|_{L^{1}}+\varepsilon^{2} \int_{0}^{t / \varepsilon}\|U(\tau)-V(\tau)\|_{L^{1}} d \tau \\
& \leq \varepsilon L\left\|U_{0}-V_{0}\right\|_{L^{1}}=L\|\bar{U}-\bar{V}\|_{L^{1}} .
\end{aligned}
$$

Finally, the continuous dependence with respect to time for solutions of (2.5) is expressed by

$$
\begin{aligned}
\left\|U^{\varepsilon}(t)-U^{\varepsilon}(s)\right\|_{L^{1}} & =\varepsilon\|U(t / \varepsilon)-U(s / \varepsilon)\|_{L^{1}} \\
& =L^{\prime}(|t-s|+\sqrt{\varepsilon}|\sqrt{t}-\sqrt{s}|) .
\end{aligned}
$$

for $t>s \geq 0$.

By Helly's Compactness Theorem, a convergent subsequence $\left\{U^{\varepsilon_{m}}\right\}_{m}$ may be extracted with $\varepsilon_{m} \downarrow 0$ as $m \rightarrow \infty$, whose limit is denoted by $U$, i.e.

$$
U^{\varepsilon_{m}}(t) \longrightarrow U(t) \quad \text { in } L_{l o c}^{1},
$$

for all $t>0$. The limit $U(t, \cdot)$ is a BV function which satisfies

$$
T V\{U(t)\}+\int_{0}^{\infty} T V\{U(\tau)\} d \tau \leq C T V\{\bar{U}\} .
$$

Moreover, by construction, $U$ is the admissible weak solution to

$$
\begin{aligned}
& U_{t}+F(U)_{x}+g(U)=H(t) \bar{U}-\int_{0}^{t} K(t-\tau) U(\tau) d \tau, \\
& U(0, x)=\bar{U}(x)
\end{aligned}
$$

when (2.5) is in conservative form, i.e. $A(u)=D F(u)$. This completes the proof of Theorem 1.

The authors in $[3,8]$ also discuss in more detail the limit to the non-conservative system and the uniqueness of solutions within the class of viscosity solutions. It would be interesting to verify these results for systems (1.5) as well.

Acknowledgments. The author would like to thank Professor Constantine M. Dafermos for suggesting this problem and helpful comments. She would also like to thank Professor Gui-Qiang Chen for his valuable remarks and discussions.

\section{REFERENCES}

[1] S. Bianchini and A. Bressan, On a Lyapunov functional relating shortening curves and viscous conservation laws, Nonlinear Anal., T.M.A. 51 (2002), 649-662.

[2] S. Bianchini and A. Bressan, A center manifold technique for tracing viscous waves, Comm. Pure Appl. Anal. 1 (2002), 161-190.

[3] S. Bianchini, A. Bresssan, Vanishing viscosity of nonlinear hyperbolic systems, Ann. of Math. 161 (2005), (1) , 223-342.

[4] A. Bressan, Hyperbolic Systems of Conservation Laws. The One Dimensional Cauchy Problem. Oxford University Press, 2000.

[5] G.-Q. Chen, C. Christoforou, Solutions for a Nonlocal Conservation Law with Fading Memory, preprint.

[6] G.-Q. Chen, C. M. Dafermos, Global solutions in $L^{\infty}$ for a system of conservation laws of viscoelastic materials with memory, J. Partial Diff. Eqs. 10 (1997), 369-383. 
[7] C. Christoforou, Hyperbolic systems of balance laws via vanishing viscosity, J. Diff. Eqs, Vol $221 / 2,470-541$.

[8] C. Christoforou, Uniqueness and sharp estimates on solutions to hyperbolic systems with dissipative source, Comm. PDE (accepted).

[9] C. Dafermos, Hyperbolic Conservation Laws in Continuum Physics, Springer-Verlag, Berlin 1999.

[10] C. M. Dafermos, Solutions in $L^{\infty}$ for a conservation law with memory, Analyse Mathematique et Applications, Gauthier-Viliars, Paris 1988.

[11] C. M. Dafermos, Solutions with shocks for conservation laws with memory. Amorphous Plymers and Non-Newtonian Fluids. (C. Dafermos, J. Ericksen and D. Kinderlehres, eds) 33-55, Springer-Verlag, New York, 1987.

[12] C. M. Dafermos, Dissipation in materials with memory, Viscoelasticity and rheology, (Madison, Wis., 1984), 221-234, Academic Press, Orlando, FL, 1985.

[13] C. M. Dafermos, Hyperbolic conservation laws with memory, Differential equations (Xanthi, 1987), 157-166.

[14] C. M. Dafermos and L. Hsiao, Hyperbolic systems of balnce laws with inhomogeneity and dissipation. Indiana Univ. Math. J.31 (1982) 471-491.

[15] C. M. Dafermos and J. A. Nohel, Energy methods for nonlinear hyperbolic volterra integrodifferential equations, Comm. PDE 4 (3) 1979, 219-278.

[16] J. Glimm, Solutions in the large for nonlinear hyperbolic systems of equations, Comm. Pure Appl. MAth. 18 (1965), 697-715.

[17] H. Hattori, S. Kawashima, Smooth shock profiles in viscoelasticity with memory, Studies in Advanced Math. 3 (1997), 271-281.

[18] P. D. Lax, Hyperbolic systems of conservation laws, Comm. Pure Appl. Math. 10 (1957), 537-566.

[19] R. C. MacCamy, An integrodifferential equation with applications in heat flow, Q. Appl. Math. 35 (1977), 1-19.

[20] R. C. MacCamy, A model for one-dimensional nonlinear viscoelasticity. Quart. Appl. Math 35 (1977), 21-33.

[21] A. Matsumura, Global existence and asymptotics of the solutions of the second-order quasilinear hyperbolic equations with the first-order dissipation, Publ. Res. Inst. Math. Sci., 13 (1977/78), pp. 349379.

[22] T. Nishida, Global smooth solutions for the second-order quasilinear wave equation with the first-order dissipation, (unpublished)

[23] J. A. Nohel, A forced quasilinear wave equation with dissipation. Proceedings of EQUADIFF IV, Lecture Notes in Math. 703, 318-327, Springer Verlag.

[24] J. A. Nohel, R. C. Rogers, A. E. Tzavaras, Weak solutions for a nonlinear system in viscoelasticity. Comm. PDE 13 (1988) no. 1, 97-127.

[25] M. Renardy, W. Hrusa and J. A. Nohel, Mathematical Problemsin Viscoelasticity, Longman, New York, 1987.

[26] D. Serre, Systems of Conservation Laws, I,II, Cambridge University Press, Cambridge, 1999.

[27] M. Slemrod, Instability of steady shearing flows in a nonlinear viscoelastic fluid, ARMA, 68 (1978), no. 3, 211-225.

[28] J. Smoller, Shock Waves and Reaction-diffusion Equations, Springer, New York, 1994.

[29] O. J. Staffans, On a nonlinear hyperbolic Volterra Equation, SIAM J. Math. Anal., 11 (1980), no. 5, 793-812.

Department of Mathematics, Northwestern University, Evanston, Illinois 60208

E-mail address: cleo@math.northwestern.edu

$U R L:$ http://www. math.northwestern.edu/ cleo 